\title{
Tests of discrete symmetries
}

\author{
M. S. Sozzi
}

Department of Physics, University of Pisa, Largo Pontecorvo 3, 56127, Pisa, IT

marco.sozzi@unipi.it

\begin{abstract}
This article provides a coarse-grained review on the experimental tests of fundamental discrete symmetries, focusing on those which appear to be deeply ingrained in Nature, or at least rooted into the description of it which we are currently capable of providing, namely those related to space-time inversions and particle/anti-particle exchange. A broad selection of tests is discussed, not in the (vain) pursuit of completeness, but rather with the aim of providing an overview of the variety of approaches, and of the underlying common principles.
\end{abstract}

Keywords: discrete symmetries, CP violation, high-energy physics

\section{Setting the scene}

As far as I see, all a priori statements have their origin in symmetry.

H. Weyl

Symmetry has since long arisen as one of the most central and fruitful concepts in physics, and as such it now holds central rôles in many experimental endeavors and theoretical speculations. It is thus mandatory to clearly state the scope of this review, and the context in which the author hopes it might have some value.

The word "tests" in the title indicates that the focus is on experimental investigations, although there is no point in trying to ignore the fruitful cross-breeding with theoretical speculations, which is briefly mentioned where needed, directing the interested reader to more detailed discussions. While "discrete symmetries" already restricts the scope significantly, some topics which would legitimately belong to such category are not addressed, such as those related to exchange symmetry and the fundamental spin-statistics connection, or some which so far only 
belong to the field of theoretical speculations, such as the breaking of (continuous) gauge symmetries down to discrete groups. The focus is on the symmetries related to space-time inversions $(P, T)$ and charge conjugation $(C)$, and their relevant combinations. Despite such restriction, no attempt to be complete was made: the field is so vast that many reviews and books exist on each individual sub-topic, and reference will be made to some of those where appropriate. Moreover, while recent results are mentioned as much as possible, in a fast-pacing field these quickly grow old, and the emphasis is not on the latest figures, but rather on concepts and approaches, since constantly updated information can be found elsewhere [1].

As for all authors who have made the effort to summarize what they know into a book, similarities to the material and approach in [2] will have to be excused, and the reader is directed there for a discussion of general and background issues.

Global discrete symmetries appear to have a less "sacred" rôle in Nature than continuous ones, both those related to space-time, through the Poincaré group, and those involving internal degrees of freedom, such as gauge symmetries, through Lie groups. Indeed, many discrete symmetries appear to be violated in some processes, although in a sufficiently limited way (since the most relevant interactions appear to respect them) to allow their very definition and recognition.

The outline of the work is as follows: symmetries are discussed in order of increasing validity, which also matches the time order in which their violations were discovered, starting from parity and charge conjugation in sections 2 and 3 , continuing with $C P$ and time reversal in sections 4 and 5, and reaching in section $6 C P T$, the only one whose validity is so far undisputed. Section 7 briefly touches on the cosmological relevance of the above symmetries, and some conclusions are presented in section 8 .

\section{Parity}

Parity $(P)$ is maximally violated by weak interactions [3], and the Standard Model of particle physics (SM) was built upon such fact. For this reason, current studies focus on testing the nature and amount of $P$ violation, with the aim of identifying possible symmetry-violating contributions beyond the SM, possibly in strong or electro-magnetic interactions.

Several systems can be investigated to probe for anomalous $P$-violating interactions, such as the recently detected coherent elastic scattering of low-energy (tens of $\mathrm{MeV}$ ) neutrinos on nuclei at small momentum transfer [4], or the scattering of low-energy polarized electrons with momentum transfers from few tens of 
$\mathrm{MeV}$ to a few $\mathrm{GeV}$ [5]; these processes are sensitive to the presence of new light mediators beyond the SM, and so far experiments do not provide indications for the existence of such particles. As an example of very low energy experimental tests, we briefly discuss atomic $P$ violation in the following.

\subsection{Parity violation in atoms}

Experiments on atomic parity non-conservation [6] search for differences with respect to the known $P$-violating interference of weak and electromagnetic interactions, to challenge the SM. $P$ violation in electromagnetic transitions between atomic levels with the same nominal parity (actually states of mixed parity due to some $P$-violating interaction) is probed by detecting the dependence of the amplitudes on the orientation of an external field. The effects due to the short-range weak interaction grow with the electron probability density in the nucleus and their velocity, both of which are proportional to the atomic number $Z$, and for this reason heavy atoms are used. The comparison to SM predictions is limited by the accuracy of the atomic calculations, which have a somewhat larger variance than the $0.35 \%$ precision of the best experiment performed on cesium at Boulder [7]; despite such theoretical uncertainties, current results appear to be compatible with the SM.

Other heavy atoms (francium, ytterbium, dysprosium, rubidium) have been probed or proposed for future experiments, either because of enhancements resulting in effects of larger magnitude, or because of more reliable theoretical calculations. The comparison of the $P$-violating effect in different isotopes, which share the same electronic structure, has been proposed to partially circumvent the uncertainties in the theoretical calculations, and is actively pursued by experiments; the variation of $P$-violating effects in different isotopes ( $\sim 1 \%$ /neutron) was recently measured in ytterbium [8].

$P$-violating effects in nuclei can give rise to $P$-odd (anapole) moments [9], which can be probed through their effect on electron scattering or the energy shifts of the low-lying electronic levels penetrating the nucleus (again growing with $Z$ ). Nuclear anapole moments can be induced by weak interaction effects in nucleon interactions, and as such represent a good probe for hadronic interactions mediated by the $Z^{0}$, since the flavour-conserving nature of weak neutral currents makes these difficult to detect against the much stronger electromagnetic and strong interactions in other systems. Again, comparisons to SM predictions are challenging, due to the usual difficulty in evaluating how strong interactions alter the weak effects in hadrons. The identification of anapole moment induced 
effects (usually a few percent of the whole $P$-violating effect) is based on their spin-dependence: while generally small, such effects can dominate over those due to $Z^{0}$ exchange in heavy atoms (see e.g. [10]). An anapole moment was detected by exploiting the comparison of the parity mixing for two different hyperfine transitions in cesium [7]: by careful comparison of the transition rates under several different reversals of experimental quantities, at different rates, systematic effects could be kept under control and a $O\left(10^{-6}\right)$ modulation detected, corresponding to a 7 standard deviation effect.

Experimental activity on $P$-violating effects in atoms is pursued at several laboratories worldwide on systems also including single atomic and molecular ions (see [6] for an extensive list of references).

\subsection{Parity and strong interactions}

While parity is verified to hold at high accuracy in strong interactions (see section 5.4, the possibility was considered that in dense and highly excited states, such as those obtained in relativistic ion collisions, local regions are formed in which $P$ is temporarily violated (see [11] and references therein). This effect might result in preferential same-charge particle emission along the system angular momentum in non-central collisions. Such asymmetries would average to zero over larger space-time regions; on the other hand, they cannot be observed on single events, due to the statistical fluctuations in the number of particles. The study of threeparticle correlations in phase space is considered to be a good probe for the above effects, which provided possible hints of their existence [12] [13] (see however [14]).

It should also be mentioned that the theoretical description of weak interactions of hadrons, for which strong interactions play a dominant rôle, presents some puzzles which require precision studies of parity-violating hadronic interactions in order to be fully clarified [15].

Most importantly, apart from weak interactions, another part of the SM Lagrangian allows for $P$ violation, namely a term involving the dual field tensor of QCD:

$$
\mathcal{L}_{C P}=\theta F_{\mu \nu}^{a} \tilde{F}^{\mu v a}
$$

where $\theta$ is a free parameter. This term violates $P$ and $C P$, and since in QFT anything which is not forbidden is mandatory (through quantum corrections), it is indeed expected to be present. Current experiments (the limits on the electric dipole moment of the neutron, see section 5.4p limit the magnitude of the effec- 
tive parameter to $|\theta| \lesssim 10^{-10}$, posing a serious fine-tuning issue, the so-called "strong CP problem" (see e.g. [16]). Possible solutions involve one of the quarks being massless ${ }^{1}$ (now excluded by lattice QCD determinations), or the existence of a new pseudo-scalar particle (the axion), so far undetected but very actively searched for, also due to its possible rôle as a dark matter candidate.

It has been remarked that a sufficiently strongly interacting dark matter axion would induce small $O\left(10^{-35} e \mathrm{~cm}\right)$ electric dipole moments in nuclei (see section 5.4), oscillating at a definite frequency ( $\sim \mathrm{kHz}$ to $\mathrm{MHz}$ ) related to the axion mass [17]; these would cause tiny $O\left(10^{-25} \mathrm{eV}\right)$ shifts in atomic energy levels, which might become detectable in the future.

\section{Charge Conjugation}

The concept of a fundamental charge-conjugation $(C)$ symmetry has its roots in special relativity, and required a long time to emerge, because the world we live in is (accidentally?) highly $C$-asymmetric.

The violation of charge conjugation symmetry by weak interactions is strictly linked to its parity violation [18], and as such built in the SM. Again, tests of $C$ symmetry focus on strong and electromagnetic interactions, using neutral systems which are $C$ eigenstates, such as the mesons $\pi^{0}, \eta, \eta^{\prime}, \omega, J / \psi$.

Decays dominated by strong interactions, such as $\eta \rightarrow 3 \pi$, were tested for the presence of $C$-violating terms, with null limits at $O\left(10^{-2}\right)$ precision from the KLOE experiment [19]. For electromagnetic decays, the best limit is $\operatorname{BR}\left(\pi^{0} \rightarrow\right.$ $3 \gamma)<3.1 \cdot 10^{-8}$ (at $90 \%$ confidence level), obtained by the Crystal Box experiment [20].

Positronium (the $e^{+} e^{-}$bound state) is a convenient system for symmetry studies in electro-magnetic interactions, as it is purely described by QED (although the relativistic nature of its constituents requires a theoretical approach for which no analytic solutions are available). Charge conjugation symmetry is verified by the absence of singlet and triplet state positronium decays into a number of photons corresponding to the opposite value of the $C$ quantum number [21] [22], the

\footnotetext{
${ }^{1}$ The effective $C P$-violating parameter in the Lagrangian gets a contribution from a possible quark mass phase.
} 
Branching Ratio limits being

$$
\begin{array}{ll}
\mathrm{BR}\left({ }^{1} S_{0} \rightarrow 3 \gamma\right)<2.8 \cdot 10^{-6} & (68 \% \mathrm{CL}) \\
\mathrm{BR}\left({ }^{3} S_{1} \rightarrow 4 \gamma\right)<3.7 \cdot 10^{-6} & (90 \% \mathrm{CL}) \\
\mathrm{BR}\left({ }^{1} S_{0} \rightarrow 5 \gamma\right)<2.7 \cdot 10^{-7} & (90 \% \mathrm{CL})
\end{array}
$$

The above limits are expected to be further improved by the J-PET experiment [23].

\section{CP}

$C P$ is the basic symmetry of weak interactions as they are built in the SM, and as such its violation is not present "by design". Actually, $C P$ is the "natural" symmetry of the gauge sector of the SM Lagrangian [24] and, as perceived by Landau [25], the one leading to the proper definition of anti-particles. Its violation is discussed at length in many reviews and several books [26] [27] [28] [2].

The group-theoretical investigation of $C P$ symmetry led to the realization that it corresponds in general to a transformation mapping the representations of all symmetries into their complex conjugates [29]; such property singles out $C P$ among discrete transformations, and implies that it cannot be broken maximally (as $P$ and $C$ are in the SM, e.g. due to the complete absence of right-handed neutrinos), since the reality of the action requires that if a complex representation appears, its complex conjugate must too. Moreover, an intriguing observation is that some discrete symmetry groups (which might be subgroups of continuous ones appearing in some models beyond the SM) are incompatible with $C P$ symmetry, thus enforcing specific values for the $C P$-violating phases ("geometrical $C P$ violation").

The (tiny) Nobel prize-worthy violation of $C P$ symmetry, discovered in 1964 through the two-pion decay of the long-lived neutral kaon [30], spoiled the attempt [25] of interpreting the handedness of space (indicated by $P$ violation) as a mere property of charge. The story of $C P$ is an intriguing one, as for more than 35 years such symmetry only appeared to be violated by neutral $K$ mesons, and in a rather peculiar way. For such reason, even after it was realized that $C P$ violation could be accommodated in the weak sector of the SM in presence of at least three quark generations [31], confirmation of such mechanism was slow to come, and alternative explanations flourished (both ad hoc, such as the super-weak ansatz [32], and generic, e.g. those involving additional Higgs multiplets). 
After many years, $C P$ violation was eventually confirmed [33] to arise (at least dominantly) from the above mechanism, related to non-trivial flavour mixing by weak interactions. The presence of a single uneliminable complex phase $e^{i \delta}$ in the Cabibbo-Kobayashi-Maskawa (CKM) mixing matrix can induce $C P$ violation in the SM. A value of $\delta$ different from 0 and $\pi$ results in the unitarity constraints for the CKM mixing matrix being represented by non-degenerate triangles (the "unitarity triangles"), whose (identical) area is the unique physical measure of $C P$ violation in the SM, expressed by the phase-definition-invariant quantity [34]

$$
J \equiv \operatorname{Im}\left(V_{i j} V_{k l} V_{i l}^{*} V_{k j}^{*}\right)=\sin \theta_{12} \sin \theta_{13} \sin \theta_{23} \cos \theta_{12} \cos ^{2} \theta_{13} \cos \theta_{23} \sin \delta
$$

where $V_{i j}$ are the elements of the CKM matrix $(i, j, k, l=1,2,3$ labeling the quark generations) and $\theta_{i j}$ the mixing angles used to parameterize them. The current determination of one such triangle is shown in figure 1; it should be remarked that by using only the constraints from observables which are not related to $C P$ violation, the existence of such phenomenon is predicted by the fit, showing the consistency of the data with the SM. The magnitude of $C P$ violation is measured to be $J=(3.18 \pm 0.15) \cdot 10^{-5}[35]$.

Since $\delta$ is a free parameter in the SM, a deeper understanding of $C P$ violation is currently lacking, and expectations are that physics beyond the SM might explain its value.

A vast worldwide research program on $C P$ violation has been ongoing for half a century, at first with the aim of elucidating in which kind of process or interaction the source of such violation arose, and later to search for further evidence of $C P$ violation in different systems, with the goal of challenging the SM description.

\subsection{The overall picture}

So far, manifestations of $C P$ violation appear to be confined to the (weak) flavour sector, coherently with the CKM mechanism which ascribes it to the single uneliminable complex phase $e^{i \delta}$ in the weak quark mixing matrix. All existing measurements are consistent with this simple picture and provide a coherent determination of the above phase angle: $\delta=(71.0 \pm 1.6)^{\circ}$ [35], which correlates in principle all $C P$-violating quantities.

While it is now clear that any new physics is not dominant in the processes which have been investigated so far, cosmological arguments (see section 7) indicate that additional sources of $C P$ violation must exist beyond the one in the $\mathrm{SM}$. The issue is thus which is the flavour structure of such new physics: if this 


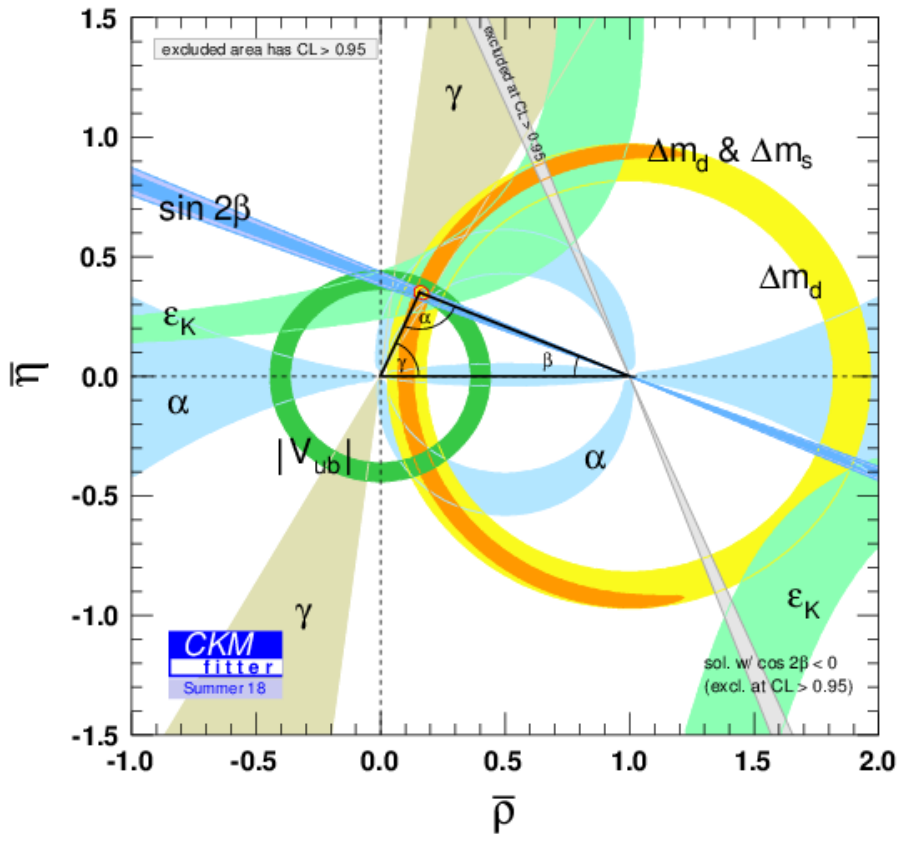

Figure 1: The present determination of one of the unitarity triangles from the measured values of various observables for $K$ and $B$ mesons. Its non-zero area is an indication of $C P$ violation, while its consistency with the triangular shape indicates that the validity of the CKM model with three quark generations. From [36]; see also [37]. 
is remarkably non-trivial, e.g. similar to that of the SM, something which would arguably call for some explanation (e.g. related to new symmetries, the "flavour problem"), this provides the suppression required to evade current experimental bounds, and the mass of the corresponding new mediator particle(s) might even be not too large compared to the electro-weak scale. Conversely, with no indication for the existence of any TeV-scale particles, it is "natural" to expect the observed suppression being due to the high mass scale of new physics, whose flavour structure is then allowed to be rather arbitrary, and wildly different from the SM. This explains why the investigation of $C P$ violation and flavour physics probes very high mass scales, up to $O(100 \mathrm{TeV})$ and above, unreachable by direct searches at colliders.

From a fundamental point of view, $C P$ violation is expressed by the presence of intrinsic complex phases in some terms of the Lagrangian; the adjective "intrinsic" is required due to the elusive nature of phases in quantum mechanics (in which the phase of a single state has no physical meaning), so that only those which cannot be eliminated by re-definition of the states are a sign of $C P$ violation. Moreover, complex phases can also be induced by $C P$-conserving interactions among particles in the initial or final state of a reaction; while such phases (called Final State Interactions (FSI) phases, or strong phases, in decay processes) can be large for hadrons or charged particles, interacting through strong or electromagnetic interactions, they are identical for particles and anti-particles, and as such do not entail $C P$ violation. Finally, the detection of $C P$-violating phases through real observables requires exploiting the interference of different amplitudes, the sub-dominant one being often suppressed by the presence of quantum loops ("penguin" diagrams), further shadowing some manifestations of $C P$ violation (see e.g. [2] for a basic discussion). We will not attempt to discuss the many ways in which $C P$ violation might be included in new physics models, on which a vast literature exists, except by pointing out that the number of uneliminable phases is naturally expected to grow with the complexity of the theory (i.e. the underlying symmetry group for gauge theories).

A violation of $C P$ symmetry would manifest most simply as a difference in some (space-integrated) property between a particle and its anti-particle, and indeed differences in partial decay rates or kinematic distributions have been investigated for this purpose. The experimental detection of the former require a precise knowledge of the relative particle/anti-particle fluxes, either from direct measurement or by exploiting associate production, while the latter can be measured independently from the normalization. Many signatures of $C P$ violation exist, and several are discussed in the following. 
$C P$ violation has been investigated in a very large number of systems, using a variety of experimental approaches, but the only evidence for such phenomenon is still limited to the meson decays in which it was originally discovered more than fifty years ago. Specifically, $C P$ violation has been detected so far in the weak decays of mesons containing quarks from different families (flavoured). No effect was measured with unflavoured mesons $\left(\pi, \eta, \eta_{c}, \ldots\right)$ : limits for such systems are given by the branching ratios of $C P$-forbidden decays such as $\eta\left(\eta^{\prime}\right) \rightarrow 2 \pi$, $4 \pi$, at varying levels of precision [35].

Tests of $C P$ symmetry have been proposed, discussed, and sometimes performed in many other systems: we mention as an example the study of momentum asymmetries in high-energy hadron collisions (see e.g. [38]) investigated at LHC experiments, with asymmetries measured in top quark pairs and $b$ hadron decays being consistent with zero and with SM predictions [39].

Before focusing in the following on flavoured mesons, we recall that $\mathrm{CP}$ violating effects in flavour-independent processes might be induced by strong $C P$ violation in the SM (section 2.2), which conversely are quantitatively irrelevant for flavoured hadron decays.

The lightest flavoured mesons with any given quark composition necessarily decay via flavour-changing weak interactions. Flavoured neutral mesons moreover exhibit a rich phenomenology, related to their flavour oscillations: two states $\left|M^{0}\right\rangle,\left|\bar{M}^{0}\right\rangle$ with opposite flavour quantum number exist, but weak interactions do not conserve flavour, thus allowing transitions among those.

$C P$ violation can manifest itself in such systems in three different ways, linked to the presence of uneliminable phases in some process.

A phase between the Hermitian and anti-Hermitian part of the effective Hamiltonian describing the meson anti-meson system results in $C P$ violation in the mixing, said to be of "indirect" type. This corresponds to the physical states not being quite $C P$ eigenstates, and rather containing components with both $C P$ eigenvalues, which induce "forbidden" decays, in the same fraction for all decay modes with the "wrong" CP eigenvalue. Expressing the two physical states as ${ }^{2}$

$$
\left|M_{a}\right\rangle=p\left|M^{0}\right\rangle+q\left|\bar{M}^{0}\right\rangle \quad\left|M_{b}\right\rangle=p\left|M^{0}\right\rangle-q\left|\bar{M}^{0}\right\rangle
$$

this kind of $C P$ violation is expressed by $|p| \neq|q|$.

A phase difference between two concurring decay amplitudes can induce $C P$ violation in the decay, said to be of "direct" type; for a neutral meson this is a

\footnotetext{
${ }^{2} C P T$ symmetry is assumed in this simplified discussion.
} 
difference between the $C P$ conjugate decay amplitudes for the processes $M^{0} \rightarrow f$ and $\bar{M}^{0} \rightarrow \bar{f}(f, \bar{f}$ being $C P$-conjugate final states).

The distinction between indirect and direct $C P$ violation is related to the origin of the symmetry violation originating in the decay process itself or rather in the (virtual) contributions to the meson oscillations which contribute to the properties of the physical states.

Finally, a difference between the phase of mixing and that of a decay amplitude results in $C P$ violation in the interference between the processes in which the neutral meson did or did not oscillate to the opposite flavour eigenstate before decaying into a final state $f$ accessible to both, i.e. between the processes $M^{0} \rightarrow f$ and $M^{0} \rightarrow \bar{M}^{0} \rightarrow f$ ("mixing-induced"). While this kind of $C P$ violation can be considered to be of either type, direct or indirect, any difference in the $C P$ asymmetry for different decay modes indicates the presence of direct $C P$ violation.

Besides the measurement of decay asymmetries between neutral mesons of different (initial) flavour, the study of the decay time distributions, which in the case of the short-lived ( $\mathrm{O}(\mathrm{ps})$ lifetimes) $B$ and $D$ mesons is allowed by the use of high-resolution vertex detectors and Lorentz-boosted particles, allows to separately measure $C P$ violation in the decay amplitudes and that related to mixing, which evolves in time.

Clearly, charged mesons, which cannot have flavour oscillations due to charge conservation, can only exhibit $C P$ violation in the decay (direct).

\subsection{The strange sector}

All three types of $C P$ violation described above were observed in neutral kaon decays, where $C P$ violation was originally discovered.

The large lifetime difference for the two neutral kaon physical states (51 ns vs. 89 ps) easily allows measurements on the long-lived $K_{L}$ to be performed: indeed such particle was the first - and for a long time only - one exhibiting $C P$ violation, through its decay into states with opposite $C P$-parity [30]. Such per mille effect turned out to be largely dominated by a $C P$-impurity in the $K_{L}$ composition (indirect $C P$ violation). The magnitude of the impurity parameter $|\epsilon|=(2.228 \pm 0.011) \cdot 10^{-3}$, related to the flavour components of the physical states by $\epsilon=(1-q / p) /(1+q / p)$, is determined from the $C P$-violating $K_{L} \rightarrow \pi \pi$ decay rates, and its real part is accurately measured by the 3 parts per thousand 
charge asymmetry in $K_{L}$ semi-leptonic decays

$$
A_{L}=\frac{\Gamma\left(K_{L} \rightarrow \pi^{-} \ell^{+} v_{\ell}\right)-\Gamma\left(K_{L} \rightarrow \pi^{+} \ell^{-} \bar{v}_{\ell}\right)}{\Gamma\left(K_{L} \rightarrow \pi^{-} \ell^{+} v_{\ell}\right)+\Gamma\left(K_{L} \rightarrow \pi^{+} \ell^{-} \bar{v}_{\ell}\right)}
$$

(where $\ell=e, \mu$ ), which is now measured in the electron mode to $2 \%$ precision (statistically-dominated) with almost 300 million events [40]. Semi-leptonic decays indeed determine the flavour of a decaying neutral kaon, since $K^{0}$ only decay to $\pi^{-} \ell^{+} v_{\ell}$ and $\bar{K}^{0}$ to the $C P$-conjugate state, to very high precision $\left(O\left(10^{-3}\right.\right.$ in the amplitudes, both in the SM and beyond, as experimentally tested); the above asymmetry therefore probes the $C P$-asymmetric composition of the physical states, while it does not get contributions from direct $C P$ violation due to the absence (or strong suppression) of interfering amplitudes.

The study of neutral kaons can be performed in different ways, depending on their production mechanism: the kaon flavour at production can be determined either by exploiting associate production through (flavour-conserving) strong interactions, using the accompanying particles in the event, or by forming a resonant state in an $e^{+} e^{-}$collider, which largely decays in entangled kaon pairs such as $K^{0} \bar{K}^{0}$, in which a flavour-specific decay of one particle of the pair determines the flavour of the other one. The former approach was used by the CPLEAR experiment at CERN [41] (1982-1996) with the exclusive processes $p \bar{p} \rightarrow K^{-} \pi^{+} K^{0}$ $\left(K^{+} \pi^{-} \bar{K}^{0}\right)$; the latter is the one used by experiments at $K$ (or $\left.\phi\right)$ factories, such as KLOE and KLOE-2 at DAФNE in Frascati [42] (2001-2018), which together accumulated $\sim 8 \cdot 10^{9} K_{S} K_{L}$ pairs (and $\sim 1.2 \cdot 10^{10} K^{+} K^{-}$pairs).

The study of neutral $K$ decays into flavour eigenstates or $C P$ eigenstates allows a diverse set of parameters to be studied; it is worth to note in passing that the entangled nature of the kaon pairs produced at $K$ factories also allows precision tests of quantum mechanical correlations.

Three generations of dedicated experiment were required to ascertain whether $C P$ violation is also present in the actual weak decay process (direct $C P$ violation): such effect shows up as a difference in the amount of $C P$ violation in different decay modes, expressed by the parameter $\epsilon^{\prime}$

$$
3 \epsilon^{\prime} \simeq \frac{A\left(K_{L} \rightarrow \pi^{+} \pi^{-}\right)}{A\left(K_{S} \rightarrow \pi^{+} \pi^{-}\right)}-\frac{A\left(K_{L} \rightarrow \pi^{0} \pi^{0}\right)}{A\left(K_{S} \rightarrow \pi^{0} \pi^{0}\right)}
$$

The non-zero measurement of such parameter by the NA48 and KTeV experiments at CERN and Fermilab [43]: $\operatorname{Re}\left(\epsilon^{\prime} / \epsilon\right)=(16.4 \pm 1.9) \cdot 10^{-4}$ eventually 
provided the first confirmation of the CKM mechanism for describing $C P$ violation in the SM. This effect corresponds to a $\sim 5 \cdot 10^{-6}$ asymmetry between the $K^{0}$ and $\bar{K}^{0}$ decay rates to $\pi^{+} \pi^{-}$.

The theoretical evaluation of the $C P$-violating parameters for kaons within the SM is very hard due to the difficulty of quantifying strong interaction effects for light hadrons, with a long-standing program of lattice QCD computations providing steady improvements. Some discrepancies with the most recent SM theoretical estimates of $|\epsilon|$ [44] appear to be related to the determination of CKM matrix elements; the theoretical precision is still one order of magnitude worse than the experimental one. A first complete lattice determination of $\operatorname{Re}\left(\epsilon^{\prime} / \epsilon\right)$ [45] also shows some disagreement with experiment, with the error being three times larger than the experimental one. Further theoretical progress is required to assess the significance of such apparent discrepancies, see e.g. [46] for a recent discussion.

The $K_{S}$ is somewhat less experimentally accessible than the $K_{L}$, and its decays are studied either through interference effects in experiments producing flavour eigenstates, or at $K$ factories, in which coherent $K_{S} K_{L}$ pairs provide tagged $K_{S}$. Limits on the $C P$-violating amplitude ratios in (neutral, unobserved) three-pion decays of $K_{S}$ are at the level $O\left(10^{-2}\right)$, almost one order of magnitude larger than the expected magnitude $\sim|\epsilon|$ [47], with a factor 2 reduction expected in the future by the analysis of the full KLOE-2 sample.

$C P$ violation in charged kaon decays was also investigated, with null results. $K^{+} / K^{-}$decay rate differences were measured with errors at the $10^{-3}$ level, while differences of Dalitz plot slopes in the abundant 3-pion decay modes were probed at the $10^{-4}$ level, with more than 3 billion events [48], showing no asymmetries, in accordance with the rough SM expectations. The current understanding that the $\mathrm{CKM}$ mechanism of the $\mathrm{SM}$ is the dominant one inducing $C P$ violation entails the presence of asymmetries also in charged kaon decays; however, the theoretical uncertainties in their evaluation, are such that the interest in these was mainly related to probing the possible presence of large non-SM effects, while modelindependent approaches indicate that the related asymmetries are expected to be small.

$C P$ symmetry was tested in hyperon decays too, by comparing the angular asymmetries due to $P$ violation in $\Lambda, \Xi^{-}, \Omega^{-}$decays to those of their anti-particles, with null results and precisions below $10^{-3}$ [35]; comparisons to theoretical predictions are even harder than for mesons.

It should be mentioned that the current frontier of physics with kaons, namely the accurate testing of the SM through the search for ultra-rare $K$ decays [49], 
is also relevant for $C P$ violation; in particular the so-far-undetected decay $K_{L} \rightarrow$ $\pi^{0} v \bar{v}$ is $C P$ violating, and allows a very clean extraction of the CKM phase, allowing a comparison with other determinations and a high sensitivity to non-SM physics; the predicted branching ratio $O\left(10^{-11}\right)$ clearly indicates the experimental difficulties of such program, which is being vigorously pursued.

\subsection{The beauty sector}

The measurement of $C P$ asymmetries in the decays of neutral $B$ mesons was the driving goal for the construction of $B$ factories at SLAC and KEK, which confirmed the belief in the CKM mechanism for $C P$ violation, eventually leading to a second Nobel prize related to $C P$ violation.

The first large $C P$ asymmetry between $B^{0}$ and $\bar{B}^{0}$ was measured in the $J / \psi K_{S}$ decay mode (branching ratio $\sim 9 \cdot 10^{-4}$ ) by the BABAR (1999-2008) and Belle (1999-2010) experiments [50]. This final state (which is $C P$-odd if the tiny $C P$ violation in $K^{0}$ mixing is neglected) is rather special because, while $B^{0}$ only decays to $J / \psi K^{0}$ and $\bar{B}^{0}$ to $J / \psi \bar{K}^{0}$ (and not vice versa), neutral kaon mixing allows both $B^{0}$ and $\bar{B}^{0}$ to reach $J / \psi K_{S}$, so that $C P$ violation in the interference of decays with and without mixing is possible. Moreover, this decay mode is dominated by a single (tree) quark-level amplitude $b \rightarrow c \bar{c} s$ (and its $C P$ conjugate), with the largest loop contributions sharing the same phase, up to corrections below $1 \%$. This implies that the value of the corresponding $C P$ asymmetry can be directly translated into the value of one angle of the unitarity triangle (corresponding to the $B^{0}-\bar{B}^{0}$ mixing phase). The first measurement was obtained in 2001, and the above asymmetry is now measured to be different from zero at 35 standard deviations, with samples of several $10^{4}$ events both from $B$-factories and collider experiments [35].

The experimental study of $B$ mesons is performed using some of the approaches described earlier for $K$ : experiments at $B$ factories [51] such as BABAR at SLAC and Belle at KEK exploit production of coherent $B$ meson pairs in (energy-asymmetric) $e^{+} e^{-}$colliders working at the $\Upsilon(4 \mathrm{~S})$ resonance to tag the neutral meson flavour, while hadron collider experiments such as $(p \bar{p})$ CDF (19852011) and D0 (1992-2011) at Fermilab [52] and ( $p p)$ LHCb at CERN [53] use statistical approaches based on the identification of the particles produced in the same event with the $B$ meson. The only approach which is not available to $B \mathrm{~s}$ is the one using physical states, since the lifetimes of the $B$ mesons are too close to allow their experimental separation at reasonable energies. 
After the existence of $C P$ violation was established in a system different from the neutral kaon, the focus shifted to the quantitative comparison with SM predictions, which is possible with sufficient precision for some $B$ decay modes (exemplified by the $J / \psi K_{S}$ one discussed above), depending on their structure in terms of quark-level amplitudes. The study of decay modes in which mixing-induced $C P$ violation asymmetries allow, either singly or in combinations, the clean extraction of parameters of the CKM matrix, ultimately allowed to tightly constrain the unitarity triangle involving $B$ mesons, showing that it is indeed remarkably consistent with being a triangle (i.e. no evidence for additional particles), and allowing the determination of its angles [37], [36], and thus the phase appearing in the CKM mixing matrix.

Besides the (tree-dominated) $b \rightarrow c \bar{c} s$ transitions, also the (tree-dominated) $b \rightarrow c \bar{u} d$ (e.g. $B^{0}, \bar{B}^{0} \rightarrow D \pi^{0}$ ) and the (penguin-dominated) $b \rightarrow s \bar{s} s$ (e.g. $B^{0}, \bar{B}^{0} \rightarrow \phi K_{S}$ ) transitions exhibit a significant suppression of contributions with a different phase, thus providing a robust handle to extract the underlying parameters; the corresponding $C P$-violating asymmetry terms are measured to 7-10 standard deviations precision. For $b \rightarrow c \bar{c} d\left(e . g . B^{0}, \bar{B}^{0} \rightarrow D^{+} D^{-}\right)$and $b \rightarrow u \bar{u} d$ (e.g. $B^{0}, \bar{B}^{0} \rightarrow \pi \pi$ ) transitions, the relative size of the sub-dominant penguin contribution is more relevant, thus introducing larger uncertainties in the extraction of the relevant parameters from the measured asymmetries, although such uncertainties can be somewhat tamed by using informations from several decay modes at once.

Similar considerations can be done for $B_{s}^{0}$ mesons, mostly studied at hadron collider experiments: the cleanest $b \rightarrow c \bar{c} s$ transition for extraction of information on the unitarity triangles is probed by the $B_{s}^{0}, \bar{B}_{s}^{0} \rightarrow J / \psi \phi$ decay mode, which was also measured, although not with a large significance so far.

$C P$ violation in the mixing of neutral $B$ mesons is expected to by small in the SM, $O\left(10^{-3} \div 10^{-4}\right)$ [54], as a result of the interplay between the virtual contributions from top quark loops and the large number of available decay channels, ultimately due to the mass pattern of the mesons. As usual, this kind of $C P$ violation is measured through charge asymmetries in semi-leptonic decays (which cannot support direct $C P$ violation), and indeed measurements from the $B$ factories indicate $C P$ violation in mixing being compatible with zero [55] to the level of $4 \cdot 10^{-4}$ on the mixing parameter, with data from the D0 collider experiment being at variance and indicating a non-zero result [56] corresponding to a mixing parameter $\operatorname{Re}(\epsilon) /\left(1+|\epsilon|^{2}\right)=(-1.24 \pm 0.43) \cdot 10^{-3}$, somewhat larger $(2.8$ standard deviations) than SM expectations (see figure 2). 


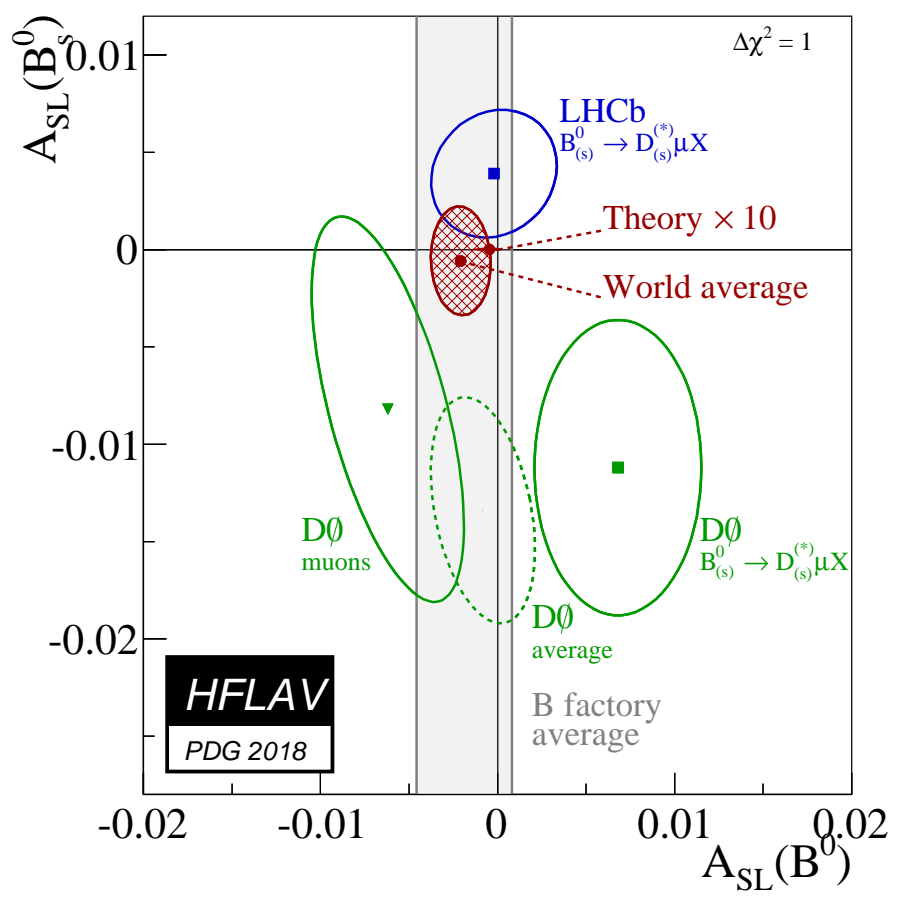

Figure 2: Current measurements of the semi-leptonic decay asymmetries for $B^{0}$ and $B_{s}^{0}$ from $B$ factories ( $B^{0}$ only) and other experiments. The red hatched ellipse shows the result of the two-dimensional averaging, taking into account the 5\% correlation between the two quantities. The red point close to the origin is the SM prediction with errors multiplied by 10 , which matches the data within 0.5 standard deviations. From [55]. 
$C P$ violation in $B_{s}^{0}-\bar{B}_{s}^{0}$ mixing was also measured by $\mathrm{LHCb}$ to be compatible with zero at the few per mille level, through the semi-leptonic asymmetries in the inclusive mode $D_{s}^{\mp} \mu^{ \pm} v X[57]$.

$B$ factories also observed direct $C P$ violation in $B$ meson decays, initially in the $B^{0} \rightarrow \pi^{+} \pi^{-}$decay mode [58] through the analysis of the decay-time dependence of the asymmetry, and later in time-integrated asymmetries for other hadronic decay modes of $B^{0}$, as well as $B^{ \pm}$. The tree-dominated decays $B^{+} \rightarrow D^{0} K^{+}$and $B^{+} \rightarrow \bar{D}^{0} K^{+}$can reach the same final states after the $D$ meson decays, and thus interfere exhibiting $C P$-violation; while in this case the weak phase appears together with the (strong) re-scattering (FSI) phase, the comparison with the $C P$-conjugate modes allows extraction of the former; kinematic analysis of multi-body final states helps to improve the precision, so that these decays ultimately provide valuable information on the unitarity triangles. Direct $C P$ violation was also detected in $B_{s}^{0}$ meson decays to $K \pi$ at the LHCb experiment [59].

Analyses of $C P$-violating contributions in the Dalitz plot distribution of threebody decays such as $B^{ \pm} \rightarrow \pi^{ \pm} \pi^{+} \pi^{-}$were also performed at $B$ factories and collider experiments, showing that the $\sim 6 \%$ integrated asymmetry exhibits kinematic structures consistent with re-scattering effects [60]; in a similar analysis of $B^{ \pm} \rightarrow$ $\pi^{ \pm} K^{+} K^{-}$LHCb measured a large $C P$-violating amplitude related to the $\pi^{+} \pi^{-} \leftrightarrow$ $K^{+} K^{-}$re-scattering [61].

Overall, hundreds of decay modes of neutral and charged $B$ mesons have been probed [35], studying both decay-time-integrated asymmetries and timedependent ones, the latter allowing identification of the contributions from different $C P$ violation sources for neutral $B$ mesons; a couple dozen $C P$-violating quantities are listed by the PDG as being established with high statistical significance. To get a flavour ${ }^{3}$ of the measured asymmetries we mention the one for $B^{+} \rightarrow f_{0}(1370) \pi^{+}$(branching ratio $<4 \cdot 10^{-6}$ ), whose central value is as large as $72 \%$, and the one for $B^{0} \rightarrow K^{+} \pi^{-}$, whose measured value $8.3 \%$ differs from zero by more than 20 standard deviations, and is measured with individual samples approaching $10^{5}$ events.

So far, all measurements are fully consistent with the origin of $C P$ violation lying in the single complex phase in the CKM mixing matrix, to which measurements of all three angles of the $B$ unitarity triangle do contribute with different significance. Further investigations aim at probing such picture at higher precision, to possibly uncover (sub-dominant) contributions from new physics; these will be pursued mostly by the LHCb experiment at CERN [62] and the Belle II

\footnotetext{
${ }^{3}$ Apologies.
} 
experiment now starting at KEK [63].

\subsection{The charm sector}

$D^{0}, D^{ \pm}$mesons, containing a charm quark and a lighter one, are the only twofamily mesons in which the heavier quark is of up-type (no mesons are formed with the top quark), and as such they exhibit large decay rates within the same family. The fact that such decays are effectively described by two quark generations only already hints to the fact that $C P$ violation effects are expected not to be large. In this respect, long-distance effects dominate: these are not enhanced by the large top quark mass and are suppressed by the magnitude of CKM elements, so that predictions for $C P$ violation in charm decays are indeed small (and somewhat uncertain) within the $\mathrm{SM}, O\left(10^{-3} \div 10^{-4}\right)$, making their investigation worthy as a probe for new physics, rather than to extract precision information on the CKM parameters.

Several classes of decays exist, corresponding to elementary amplitudes of different magnitude, according to the CKM matrix elements involved: $c \rightarrow u s \bar{d}$ ("favoured"), such as $D^{0} \rightarrow K^{-} \pi^{+}, c \rightarrow u d \bar{d}(s \bar{s})$ ("singly Cabibbo suppressed"), such as $D^{0} \rightarrow \pi^{+} \pi^{-}\left(K^{+} K^{-}\right)$, and $c \rightarrow u d \bar{s}$ ("doubly Cabibbo suppressed"), such as $D^{0} \rightarrow K^{+} \pi^{-}$.

The flavour oscillations of neutral charmed mesons were only detected in 2007 at $B$ factories, since the mass and width differences of the two physical states which drive their frequency are only $O\left(10^{-2}\right)$ relative to their average decay width. The investigation of $C P$ violation related to such process (either in the mixing or in the interference of mixing and decay) is performed by studying the nonexponential rate dependence of the decays, to extract quantities such as the relative decay asymmetry of $D^{0}$ and $\bar{D}^{0}$ into $K^{+} K^{-}$states, which is so far measured to be consistent with zero with an error of order a few $10^{-4}$. A fit of neutral $D$ meson decay parameters gives no indication of a $C P$ asymmetry in the composition of the physical states, at present with an error of several percent.

After a first hint of $C P$ violation in the hadronic decays of neutral charmed mesons was reported in 2011, the $\mathrm{LHCb}$ experiment eventually obtained in 2019 the first evidence for $C P$ violation in the decays of $D^{0}, \bar{D}^{0}$ mesons to hadrons [64]. The singly-Cabibbo-suppressed decay modes $D^{0} \rightarrow \pi^{+} \pi^{-}\left(K^{+} K^{-}\right)$were studied, in which the flavour of the neutral $D$ meson is tagged by the charge of accompanying particles in its parent decay, namely the pion in $D^{*}(2010)^{+} \rightarrow D^{0} \pi^{+}$or the muon in $\bar{B} \rightarrow D^{0} \mu^{-} \bar{v}_{\mu} X$. The experiment measured the difference between the decay-time- 


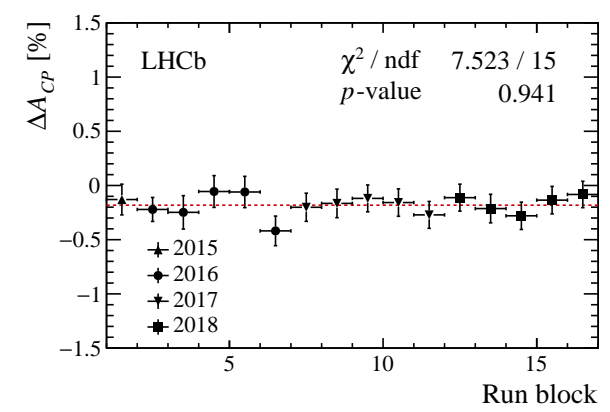

(a) Pion-tagged sample.

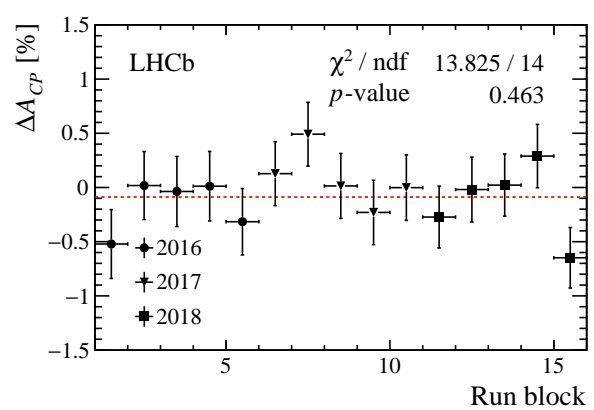

(b) Muon-tagged sample.

Figure 3: Measurements of the difference between $C P$ asymmetries to $K^{+} K^{-}$and $\pi^{+} \pi^{-}$decays in time-ordered data-taking sub-samples, with statistical uncertainties only. The horizontal red-dashed lines show the central values of the nominal results. From [64].

integrated asymmetries for the two decay modes above, in which the experimental detection asymmetry (related to the charge-dependent detection efficiencies for the flavour tagging particles) and the production asymmetry (related to the initial state charge asymmetry in a $p p$ collider) do cancel.

The result obtained with samples of several $10^{7}$ decays is

$$
A_{C P}\left(K^{+} K^{-}\right)-A_{C P}\left(\pi^{+} \pi^{-}\right)=(-15.4 \pm 2.9) \cdot 10^{-4}
$$

which is more than 5 standard deviations from zero and, being a difference between $C P$ violation in different decay modes, indicates the presence of direct $C P$ violation (see figure 3). The assessment of the compatibility of this result with the SM predictions requires further improvements in the theoretical precision.

The decay asymmetries of charmed hyperons were also tested for $C P$ asymmetries at the percent level, with null results [35].

\subsection{Leptons}

The evidence for neutrino oscillations opened the way to studies of $C P$ violation in the leptonic sector, and indeed experiments do not deal with bound states, nor with transitions among charged leptons, but rather with asymmetries in neutrino oscillation probabilities.

The Pontecorvo-Maki-Nagakawa-Sakata (PMNS) mixing matrix [65] is the analogous of the CKM matrix, and thus the presence of three generations of neu- 
trinos, with all masses and mixing angles $\theta_{i j}$ known to be different from zero [35], allows the presence of a single non-trivial $C P$-violating phase $e^{i \delta}$ (arguably unrelated to the one in the CKM matrix, despite the use of the same notation). In this scenario, just as for quarks, all $C P$-violating asymmetries are proportional to the unique rephasing-invariant quantity $J$ expressing the magnitude of symmetry breaking:

$$
J=\frac{1}{8} \cos \theta_{13} \sin 2 \theta_{12} \sin 2 \theta_{23} \sin 2 \theta_{13} \sin \delta \simeq(0.026 \div 0.035) \sin \delta
$$

One possible difference with respect to the quark case is suggested by the (so far undisputed) evidence for lepton family number conservation in charged leptons: neutrinos might be Majorana particles, identical with their anti-particles. In such case two more $C P$-violating phases are present (for three neutrinos, while one would be present even for two neutrinos), although these do not influence the neutrino mixing probabilities, and as such are only probed by processes such as neutrinoless double beta decay $(A, Z) \rightarrow(A, Z+2)+2 e^{-}$.

The decay of pions produced in accelerators and the interaction of cosmic rays in the atmosphere are sources which can be used in experiments to study neutrinos and anti-neutrinos.

By comparing two neutrino flavour transition probabilities, several discrete symmetries can be tested, i.e. $C P$ by comparing $v \rightarrow v^{\prime}$ and $\bar{v} \rightarrow \bar{v}^{\prime}$, or $T$ (section 5) by comparing $v \rightarrow v^{\prime}$ and $v^{\prime} \rightarrow v$. CPT symmetry (section (6) is probed instead by comparing $v \rightarrow v^{\prime}$ and $\bar{v}^{\prime} \rightarrow \bar{v}$, and its validity would be expected to enforce the equality of $C P$ - and $T$-violating asymmetries.

The tiny cross-sections and long oscillation wavelengths for neutrinos imply that in experiments these particles usually travel long distances through matter (including the earth, or the sun in the case of solar neutrinos); this introduces an environmental $C P$ (and $C P T$ ) asymmetry between neutrinos and anti-neutrinos [66] which in general alters the experimental signatures due to $C P$ and $T$ violation effects. Disentangling such "matter effects" from genuine $C P$ violation requires adequate modeling of the interactions (see e.g. [67]) and the use of additional information, such as the energy dependence of the oscillation probabilities [68].

The study of $v_{\mu} \rightarrow v_{e}$ and $\bar{v}_{\mu} \rightarrow \bar{v}_{e}$ oscillations with muon neutrino (or antineutrino) beams of $\sim \mathrm{GeV}$ energy produced at proton accelerators was performed most recently by the long-baseline experiments T2K in Japan and NovA in the USA. T2K [69] analyzed an integrated flux corresponding to $2.2 \cdot 10^{21}$ Protons On Target (POT) at a distance of $295 \mathrm{~km}$ from production, and $\mathrm{NO} v \mathrm{~A}$ [70] a flux corresponding to $8.9 \cdot 10^{20}$ POT at a distance of $810 \mathrm{~km}$, studying both the ap- 
pearance of $v_{e}, \bar{v}_{e}$ and the disappearance of $v_{\mu}, \bar{v}_{\mu}$ to extract information on the $C P$-violating phase $\delta$. Both experiments report best fit values for $\delta$ which disfavour $C P$-conserving values at the level of 1-2 standard deviations.

The Super-Kamiokande detector used by the T2K experiment has also been long used to study atmospheric neutrinos (and anti-neutrinos), by measuring the electron and muon neutrino survival probabilities and the $v_{\mu} \leftrightarrow v_{e}$ oscillations, mainly through the analysis of the zenith angle distribution which distinguishes particles which crossed the earth from those who did not. The best fit to the data corresponding to a mass exposure of 328 kton years also gives a best fit value of $\delta$ slightly favoring $C P$ violation [71].

Overall, the average [35] of the above experiments gives a value for the $C P$ violating phase $\delta=\left(2.74_{-0.32}^{+0.36}\right) \pi / 2$, compatible $\left.\right|^{4}$ with the maximal $C P$-violating case $\delta=3 \pi / 2$, and disfavoring $C P$ conservation at $\sim 2$ standard deviations, still far from solid evidence of $C P$ violation. For the above central value of the phase, the corresponding magnitude of the $J$ invariant would be close to -0.03 , three orders of magnitude larger than in the case of quarks.

The measurement of the leptonic $C P$-violating phase is central in the program of future envisaged long-baseline neutrino experiment, planned to start datataking in the second half of 2020s. Clearly, the precision to which $\delta$ can be measured depends on its value, but estimates for the long-baseline DUNE experiment in the USA [72] (40 kton liquid-argon detector exposed to neutrinos produced by a $1 \mathrm{MW}$ proton beam $1300 \mathrm{~km}$ away) and the Tokai-to-HyperKamiokande experiment in Japan [73] (380 kton water Čerenkov detector exposed to neutrinos produced by a $1.3 \mathrm{MW}$ proton beam $300 \mathrm{~km}$ away) indicate that - for the currently favoured values of mixing parameters - such phase could be determined with a 3 standard deviation significance for $75 \%$ of its possible values, over the 10-20 years time duration of the experiments.

For what concerns charged leptons, the coefficient of an effective $C P$-violating term in their interaction with photons can be constrained by an analysis of the kinematical polarization-dependent distributions In the case of the $e^{+} e^{-} \rightarrow \tau^{+} \tau^{-}$ process, by fitting different $\tau$ decay modes, determining the $\tau$ spin direction from momenta (assuming massless electrons and neutrinos, and empirically averaging over unmeasurable kinematic variables), the lowest-order dependence of the cross section upon the above coefficient allows its extraction. The real part of the coefficient which does not depend on the centre of mass energy can be interpreted

\footnotetext{
${ }^{4}$ The results actually depend on the currently unknown mass ordering of the neutrino states, the quoted one being that for the so-called "normal" mass ordering.
} 
as the $T$-odd electric dipole moment of the $\tau$ lepton, $d_{\tau}$. By analyzing 30 million events, the Belle collaboration obtained [74]

$$
\begin{aligned}
& -2.2 \cdot 10^{-17} e \mathrm{~cm}<\operatorname{Re}\left(d_{\tau}\right)<4.5 \cdot 10^{-17} e \mathrm{~cm} \\
& -2.5 \cdot 10^{-17} e \mathrm{~cm}<\operatorname{Im}\left(d_{\tau}\right)<0.8 \cdot 10^{-17} e \mathrm{~cm}
\end{aligned}
$$

(at 95\% confidence level), consistent with $C P$ symmetry. This result improves on earlier limits obtained at the CERN LEP $e^{+} e^{-}$collider, running at the centre of mass energy of the $Z^{0}$ boson, in which contributions from weak interactions were dominant.

Further insight on the effects of leptonic $C P$ violation can be obtained from dedicated reviews, e.g. [75].

\section{Time reversal}

Due to the peculiar nature of time in physics, the violation of time reversibility has long been a subject of deep study. The most evident manifestations of such violation, namely the macroscopic irreversibility (the thermodynamic arrow of time) and the large-scale evolution of the universe (the cosmological arrow of time) seem to be related to "boundary" conditions (in a rather wide sense) rather than to fundamental laws, and the symmetry with respect to microscopic time reversal has apparently no direct connection to those.

Focusing on microscopic time reversal, it should be mentioned that even the definition of such operation raises fundamental issues [76], as for all transformations which cannot be operationally performed in an active way, and even more so in this case [77]. Leaving considerations of fundamental nature aside, the question of whether known interactions are invariant under the time reversal operation $T$ has been posed and studied with different approaches (see e.g. [78], [6]).

\subsection{Indirect tests}

Indirect evidence for the violation of $T$ in weak interactions has been known for a long time, through the phenomenon of $C P$ violation in the mixing of neutral kaons (section 4.2). Clearly, by assuming the validity of $C P T$ symmetry, any evidence of $C P$ violation implies $T$ violation, and in this sense the SM (being a quantum field theory which satisfies the $C P T$ theorem) definitely includes $T$ violation. It is important to note that such an assumption is not required, however: after the 
shock caused by the fall of $C P$ symmetry, physicists became rather cautious with undue assumptions, and considered the most general form for any interaction. The (weak-interaction-driven) effective Hamiltonian for $K^{0}-\bar{K}^{0}$ mixing can be parameterized in full generality, allowing $C P$-violating terms which also violate either $T$ or $C P T$, and the data unambiguously indicates that $C P T$ symmetry is valid (and therefore $T$ violated) to a very high level of precision (section 6.2). To the best of our present knowledge, the magnitudes of $C P$ and $T$ violation are identical.

As mentioned, a study of $T$ violation with neutrinos might be performed by comparing oscillation probabilities between neutrino flavours, taking into account the fact that matter effects introduce effective differences with respect to $C P$-violating asymmetries, even if $C P T$ symmetry holds. $T$-symmetry tests using electron and muon neutrinos (or anti-neutrinos) are conceivable, but lacking sources of high-energy electron neutrinos (for which flavour identification is possible), such tests would require either novel electron neutrino sources (such as "beta beams" of boosted radioactive ions) [79] or the exploitation of the electron neutrinos obtained by flavour oscillations themselves [80], both being ideas for future projects.

\subsection{T-odd tests}

Subtleties linked to the fact that time reversal is described by an anti-unitary transformation operator imply that - contrary to the case of $P$ - the measurement of a non-zero average value for a quantity changing sign under $T$ ( $T$-odd) is not conclusive evidence for $T$ violation. The reason boils down to the fact that comparing the properties of a process with those of its time-reversed version is not really possible in practice when using free quantum particles, as this would require exchanging initial and final states, which have different asymptotic behaviour. What is usually considered are rather $T$-odd quantities, which change sign when momenta and spins are reversed (sometimes called "naive" time reversal), but it should be kept in mind that $T$-conserving interactions can generate non-zero values for such quantities. Only when independent quantitative information is available on the magnitude of such interactions, definite conclusions can be reached on $T$ violation from the measurement of a non-zero $T$-odd quantity (i.e. in case it can be shown that interactions cannot possibly induce an effect as large as the one being observed). All the results from tests of this type are so far consistent with $T$ symmetry. 
Scalar $T$-odd quantities studied in particle physics involve at least three independent momentum or spin vectors combined in a triple product, the simplest example being $\boldsymbol{S}_{i} \cdot \boldsymbol{p}_{j} \times \boldsymbol{p}_{k}$ (the polarization of particle $i$ transverse to the plane defined by momenta $j, k$ ). In practice one can consider the transverse polarization of particles in (non-collinear, two-body) scattering experiments or, conversely, the scattering distribution for polarized particles; since scattering experiments are usually performed with particles interacting mainly through strong or electromagnetic interactions, this restricts the above tests to such interactions, while at the same time introducing the possibility of large spurious effects, thus limiting their potential reach. $T$ tests involving the elastic scattering of electron neutrinos on a polarized electron target have been proposed [81]: by measuring the angular distribution of electrons scattered by an intense $\sim 1 \mathrm{MeV}$ neutrino radioactive source decaying by electron capture, such experiments might be sensitive to $T$-violating effects beyond the SM, but significant technical developments are required to realize them.

In (weak) decay processes, the interaction among final state particles is the main source of possible spurious effects inducing non-zero average values of $T$ odd quantities, although it should be remarked that any on-shell intermediate state contributing to the decay and generating a phase can generate such an effect, and this can be relevant in some cases [82].

In a decay process, the independence of the two momentum vectors in the $T$ odd product considered above requires a decay involving at least three particles in the final state. Examples with a long history are the $D$ and $R$ parameters in beta decay, which parameterize the dependence of the decay rate on $\boldsymbol{J} \cdot \boldsymbol{p}_{e} \times \boldsymbol{p}_{v}$ and $\boldsymbol{\sigma} \cdot\left(\boldsymbol{J} \times \boldsymbol{p}_{e}\right)$ respectively, $\boldsymbol{J}, \boldsymbol{\sigma}$ being the nuclear and electron spin vectors, respectively. The experimental measurement of the above quantities requires polarized nuclei (or neutrons) and the detection of the neutrino momentum through that of the recoil nucleus (or proton), or the transverse electron polarization, both of which are not easy to obtain. While measurements on neutrons are free from any uncertainties linked to nuclear structure, the long neutron lifetime introduces different experimental challenges. The magnitude of $D(R)$ induced by $C P$ violation in the SM is $\sim 10^{-12}\left(\sim 10^{-14}\right)$ [83], and final state interactions induce effects of magnitude at most $\sim 10^{-5}\left(\sim 10^{-3}\right)$. Since $D$ is $P$-even and $R$ is $P$ odd, these parameters are somewhat complementary in probing physics beyond the SM. Measurements by the emiT-II and nTRV experiments show no evidence of $T$ violation, with errors of order few $10^{-4}\left(10^{-2}\right)$ on $D(R)$, dominated by statistics [84], [85]. Measurements of the $D$ coefficient in hyperon decays were also performed, but the precision is much lower in this case. 
Similar tests search for the $T$-odd muon polarization transverse to the decay plane in semi-leptonic weak decays such as $K_{L} \rightarrow \pi^{+} \mu^{-} \bar{v}$ and $K^{+} \rightarrow \pi^{0} \mu^{+} \nu$. In these decays not involving nuclei the spurious effects due to FSI are smaller, thus allowing more significant tests. The limit at which spurious SM FSI start contributing was reached for the $K_{L}$ decay mode, while that of $K^{+}$(with a single charged particle in the final state) is still an open subject of investigation: the known $C P$ violation in the SM induces effects of order $10^{-7}$ in the transverse polarization $P_{T}$, and FSI effects can reach $10^{-5}$, while the present experimental precision is at the level of few $10^{-3}$. As precision increases, these experiments require more careful design to maintain a matching control of the systematic effects. The most recent null result is from the E246 experiment at KEK [86], with a statistically-limited error $2.5 \cdot 10^{-3}$ on $P_{T}$. A proposal was put forward years ago [87] for a new experiment, requiring an upgraded J-PARC accelerator, to reach the $10^{-4}$ level.

A similar test can be performed in $\mu^{+}$decay: since neutrinos are not detected, this requires searching for a $T$-odd polarization of the positron transverse to the plane defined by the muon spin and the electron momentum, thus requiring polarized muons. The advantage of using a leptonic decay is the smallness of spurious FSI effects. The best limit was obtained by a PSI experiment [88] exploiting the precession of stopped $\mu^{+}$and measuring the polarization of the decay $e^{+}$through the angular distribution of the photons resulting from its annihilation on polarized electrons: the statistically-dominated error is $8.4 \cdot 10^{-3}$.

Tests are also performed in systems mostly governed by QED, such as orthopositronium decay: within the SM, the rate dependence on the $T$-odd and $P$ odd correlation $\left(\boldsymbol{S} \cdot \boldsymbol{k}_{1}\right)\left(\boldsymbol{S} \cdot \boldsymbol{k}_{1} \times \boldsymbol{k}_{2}\right)$, where $\boldsymbol{S}$ is the positronium spin and $\boldsymbol{k}_{1,2}$ the two larger photon momenta, is only due to photon-photon FSI at the level $O\left(10^{-10}\right)$; the measurement is compatible with zero with a statistically-dominated error $\sim 2 \cdot 10^{-3}$ [89]. The quantity $\boldsymbol{S} \cdot \boldsymbol{k}_{1} \times \boldsymbol{k}_{2}$ is $T$-odd and $P$-even (and as such also $C P T$-odd), and was also measured to be zero with an error $\sim 3 \cdot 10^{-3}$ [90]. One of the main challenges in such experiments is the determination of the positronium polarization, for which improvements are possible in the future. Possibilities also include measurements of asymmetries involving the photons' polarizations, such as $\boldsymbol{S}_{1} \cdot \boldsymbol{k}_{2}$, the $T$-odd (and $C P T$-odd) scalar product of the polarization of the most energetic photon and the momentum of the second most energetic one [91].

Scalar $T$-odd quantities can be formed which do not require polarization measurements, and are therefore less experimentally challenging. These involve the triple product of three independent momenta in a decay involving at least four particles in the final state; one can consider the radiative version of the decays men- 
tioned above, with smaller rates, thus trading statistics for systematics. Indeed, a $T$-odd correlation which was studied is $\boldsymbol{p}_{\gamma} \cdot\left(\boldsymbol{p}_{\pi} \times \boldsymbol{p}_{\ell}\right)$ in $K^{ \pm} \rightarrow \pi^{0} \ell^{ \pm} v \gamma(\ell=e, \mu)$ decay [92]. FSI effects on the relative asymmetries for events with positive or negative values of the above correlations were estimated to be $O\left(\right.$ few $\left.10^{-4}\right)$ [93]. An experimental measurement of such asymmetry was obtained for $K^{-} \rightarrow \pi^{0} \ell^{-} \bar{\gamma} \gamma$ decay by the ISTRA + experiment at Protvino [94]: it is consistent with zero with an error of $2 \cdot 10^{-2}$. Improvements by an order of magnitude can be expected from higher statistics kaon experiments. An interesting possibility is the comparison of the above asymmetries for $K^{+}$and $K^{-}$decays: these are both experimentally accessible, and systematic differences between the two (which would be a limit for low-energy polarization measurements) can be controlled in high-energy experiments. Spurious FSI effects cancel in the difference of the $T$-odd asymmetries for the two charge modes, which therefore allows a clean test of $T$ symmetry.

The asymmetry between positive and negative values of the triple product $\boldsymbol{p}_{K^{+}}$. $\left(\boldsymbol{p}_{\pi^{+}} \times \boldsymbol{p}_{\pi^{-}}\right)$was measured in the decays of $D^{+}$and $D_{s}^{+}$to $K^{+} K_{S} \pi^{+} \pi^{-}$, as well as $D^{0}$ to $K^{+} K^{-} \pi^{+} \pi^{-}$, four-body decays with sizeable branching ratios $\left(10^{-3}\right.$ to $\left.10^{-2}\right)$; the difference to the corresponding asymmetry for the charge-conjugate decays of $D^{-}, D_{s}^{-}, \bar{D}^{0}$ is a genuine signal of $T$ violation. While the above quantities are expected to be tiny in the SM [95], they could receive large contributions from new physics and can be used to search for it. The analysis of samples of some $10^{4}$ decays by BABAR resulted in asymmetries in the $1-10 \%$ range, whose differences are compatible with zero to an absolute precision in the $0.5-1 \%$ range [96].

A similar $T$-odd correlation involving only momenta, $\boldsymbol{p}_{\gamma} \cdot\left(\boldsymbol{p}_{e} \times \boldsymbol{p}_{v}\right)$ in beta decay was proposed for investigation [97]; FSI effects are estimated to be of order $10^{-4}\left(10^{-5}\right)$ for neutron (nuclear) radiative beta decays.

A non-zero $T$-odd decay plane asymmetry $(13.7 \pm 1.5) \cdot 10^{-2}$ was measured in the rare decay $K_{L} \rightarrow \pi^{+} \pi^{-} e^{+} e^{-}$(branching ratio $\simeq 3 \cdot 10^{-7}$ ), the value being fully consistent with that induced by (indirect) $C P$ violation in $K_{L} \rightarrow \pi^{+} \pi^{-}$decay (no asymmetry was measured in the decay of the $K_{S}$ to the same final state, as expected). This measurement illustrates a general feature of the $e^{+} e^{-}$conversion process, providing experimental access to the polarization of a virtual photon, thus allowing to probe $C P$ - and $T$-odd quantities which would average to zero when integrating over the (unmeasurable) photon polarization. A similar approach was used in the analysis of the decay $K_{L} \rightarrow e^{+} e^{-} e^{+} e^{-}$, where no significant $C P$ asymmetry was measured [35]. In the non-flavour-changing decay $\eta \rightarrow \pi^{+} \pi^{-} e^{+} e^{-}$, for which the contribution from SM $C P$ violation is entirely negligible $O\left(10^{-15}\right)$ [98], the measurement is consistent with $C P$-conservation at the $3 \cdot 10^{-2}$ level [99]. 
$T$-odd correlations in top-quark pair production and decays ([100] and references therein) can be investigated at the LHC: by using the momenta of the decay particles (or jets), asymmetries which probe $C P$ can be formed, despite the $C P$ asymmetry of the initial $p p$ state, because the production mechanism is dominated by gluon or $q \bar{q}$ processes.

\subsection{Direct tests}

Strictly speaking, direct tests of time reversal symmetry are ultimately hindered in the quantum world by the impossibility of reversing asymptotic states in a nonstationary (e.g. decay) process.

The so-called Kabir asymmetry [101] quantifies the difference between the rates of two time-reversed processes, namely the transition probabilities between neutral flavoured mesons $M^{0}$ (i.e. $K^{0}, D^{0}, B^{0}$ ), namely:

$$
A_{T}=\frac{P\left(\bar{M}^{0} \rightarrow M^{0}\right)-P\left(M^{0} \rightarrow \bar{M}^{0}\right)}{P\left(\bar{M}^{0} \rightarrow M^{0}\right)+P\left(M^{0} \rightarrow \bar{M}^{0}\right)}
$$

Since the physical states (with definite lifetimes) are linear combinations of $M^{0}, \bar{M}^{0}$, the above asymmetry can be actually measured by tagging the flavour of a meson at production time and at decay time. This was done for the first time with neutral kaons by the CPLEAR experiment at CERN [102]. Such experiment exploited the exclusive reactions $p \bar{p} \rightarrow K^{ \pm} \pi^{\mp} K^{0}\left(\bar{K}^{0}\right)$, to tag the strangeness of the neutral kaon at the time of production (by strong interactions) by the charge of the accompanying kaon. The strangeness content of the neutral kaon then evolved in time due to weak-interactions, and it was determined at the time of decay by considering semi-leptonic decays $K^{0} \rightarrow e^{+} \pi^{-} v_{e}\left(\bar{K}^{0} \rightarrow e^{-} \pi^{+} \bar{v}_{e}\right)$, through the charge of the emitted electron 5 . The measured asymmetry at time $t$ after kaon production

$$
\frac{P\left(\bar{K}^{0} \rightarrow e^{+} \pi^{-} v_{e} ; t\right)-P\left(K^{0} \rightarrow e^{-} \pi^{+} \bar{v}_{e} ; t\right)}{P\left(\bar{K}^{0} \rightarrow e^{+} \pi^{-} v_{e} ; t\right)+P\left(K^{0} \rightarrow e^{-} \pi^{+} \bar{v}_{e} ; t\right)}
$$

was found to be time-independent (as expected in absence of decays violating the “ $\Delta S=\Delta Q$ " rule) with an average value $(6.6 \pm 1.6) \cdot 10^{-3}$ [102], different from zero

\footnotetext{
${ }^{5}$ The opposite charge combination only arises in the SM through second-order weak interactions, thus being suppressed by $O\left(10^{-6}\right)$.
} 
at the level of 4 standard deviations. Such asymmetry coincides with the above $A_{T}$.

Clearly, since $M^{0}$ and $\vec{M}^{0}$ are $C P$-conjugate states, the Kabir asymmetry cannot distinguish between $C P$ and $T$ symmetry (i.e. a non-zero $A_{T}$ violates both, and conversely if either such symmetry were valid it would force $A_{T}$ to vanish). Questions were raised [103] on the interpretation of $A_{T}$ as a genuine manifestation of $T$ violation, because such asymmetry appears to be intrinsically linked to a nontime-reversible decay process, but it was later shown [104] that this is not an issue, and that indeed the CPLEAR result provided direct evidence for the violation of time reversal symmetry (without assumptions on CPT symmetry [105]).

The Kabir asymmetry was also measured in quite the same way for neutral $B$ mesons, using several approaches for initial flavour tagging, and semi-leptonic decays such as $B^{0} \rightarrow D^{-} \mu^{+} v$ (and the corresponding $C P$-conjugate mode) or the corresponding one with a $D^{*}$ charged meson in the final state. As mentioned, measurements of such asymmetries are so far consistent with zero. Alternatively, the same quantity can be obtained by measuring the charge asymmetry for like-sign di-lepton decays, i.e. $\left(N^{++}-N^{--}\right) /\left(N^{++}+N^{--}\right)$, where $N^{++}\left(N^{--}\right)$is the number of events with two positive (negative) leptons in the final state, consistent with originating from the decay of a $B^{0} \bar{B}^{0}$ meson pair. Apart from the measurement of an asymmetry in like-sign di-lepton decays by the D0 experiment [56], all other measured asymmetries, from $B$ factories and hadron colliders, are consistent with $T$ (and $C P$ ) symmetry [35], with errors of order few $10^{-3}$, both for $B^{0}$ and $B_{s}^{0}$ mesons.

A different approach was proposed [106] to measure at $B$ factories a $T$-violating asymmetry which does not relate $C P$-conjugate states (i.e. one which would not be forced to vanish in case $C P$ symmetry would hold). Considering decays to $C P$ eigenstates $f_{ \pm}$(both accessible from $B^{0}$ as well as from $\bar{B}^{0}$ ) one can define a pair of (neutral $B$ meson) states $B_{ \pm}$, with $B_{+}$being the linear combinations of flavour eigenstates $B^{0}, \bar{B}^{0}$ which can only decay to $f_{+}$, and not to $f_{-}$(and vice versa). The choice $f_{+}=J / \psi K_{L}$ and $f_{-}=J / \psi K_{S}$ (which are $C P$ eigenstates if the tiny $C P$ violation in $K^{0}$ mixing is neglected) is peculiar: first, these states are indeed of the type described above, accessible from both $B^{0}$ and $\bar{B}^{0}$ thanks to $K^{0}-\bar{K}^{0}$ mixing, despite the fact that (neglecting possible violations of the " $\Delta B=\Delta Q$ " rule) $B^{0}$ only decays to $J / \psi K^{0}$ and $\bar{B}^{0}$ to $J / \psi \bar{K}^{0}$ (and vice versa). Second, such decays are almost completely dominated by elementary amplitudes with a single phase (section 4.3), and therefore CPT symmetry implies that the corresponding decay 
amplitudes for the flavour eigenstates $A\left(B^{0} \rightarrow J / \psi K^{0}\right), A\left(\bar{B}^{0} \rightarrow J / \psi \bar{K}^{0}\right)$ are equal in magnitude, which results in $B_{+}$and $B_{-}$being orthogonal states. Now, the initial $B$ meson pair can be expressed in terms of $B_{ \pm}$states (which are $C P$ eigenstates if $C P$ violation in kaon mixing is neglected), and its entangled nature is such that when one meson is observed to decay to e.g. $J / \psi K_{S}$, the other one is known to be in the $B_{+}$state at that same time. In this way, detecting the (later) decay of the second meson to another state, asymmetries can be formed such as

$$
\frac{P\left(B^{0} \rightarrow B_{-}\right)-P\left(B_{-} \rightarrow B^{0}\right)}{P\left(B^{0} \rightarrow B_{-}\right)+P\left(B_{-} \rightarrow B^{0}\right)}
$$

and three similar ones, whose non-zero value only violates $T$ symmetry.

When sufficient statistics became available, the BABAR experiment performed the above study [107], which requires analyzing events according to whether their flavour-tagging decay occurs before or after the $C P$-tagging decay of the companion meson. All four $T$-violating time-dependent asymmetries were measured, obtaining evidence of $T$ violation with a significance corresponding to 14 standard deviations (figure 4). Some $C P$ - and $C P T$-violating asymmetries were also extracted, providing independent confirmation for the validity of $C P T$ symmetry and the fact that the measured $T$ violation is fully consistent with what is expected from $C P$ violation only.

Similar measurements might in principle be obtained for $K^{0}$ at a $e^{+} e^{-}$kaon factory running at the $\phi$ resonance (e.g. at DA $\Phi N E)$, provided enough luminosity is available [109], as well as for $D^{0}$ at a factory running at the $\psi(3770)$, and for $B_{s}^{0}$ at the $\Upsilon(5 \mathrm{~S})$.

Note that the above approach cannot provide a $T$-violating measurement for any pair of states: by measuring the decay to a final state $f$ of one meson in the entangled pair, such meson is filtered to be (at the decay time) in the state orthogonal to the one which cannot decay to $f$, and such state is not in general the only one which can decay to some other final state $g$, except in some special cases as those mentioned above ( $C P$-conjugate states $B^{0}, \bar{B}^{0}$ or opposite $C P$ eigenstates with the same flavour content $\left.B_{+}, B_{-}\right)$. A proposed alternative approach [110] considers the fact that a connection to experimentally measurable quantities can be obtained if the survival probability of the state is also measured, requiring an inclusive decay measurement, or if three decay channels are considered together.

As mentioned, $T$ violation parallels $C P$ violation if $C P T$ symmetry is valid: referring to the classification of $C P$ violation in weak decays, we recognize that just as the Kabir asymmetry is evidence for $T$ violation in neutral meson mixing, 


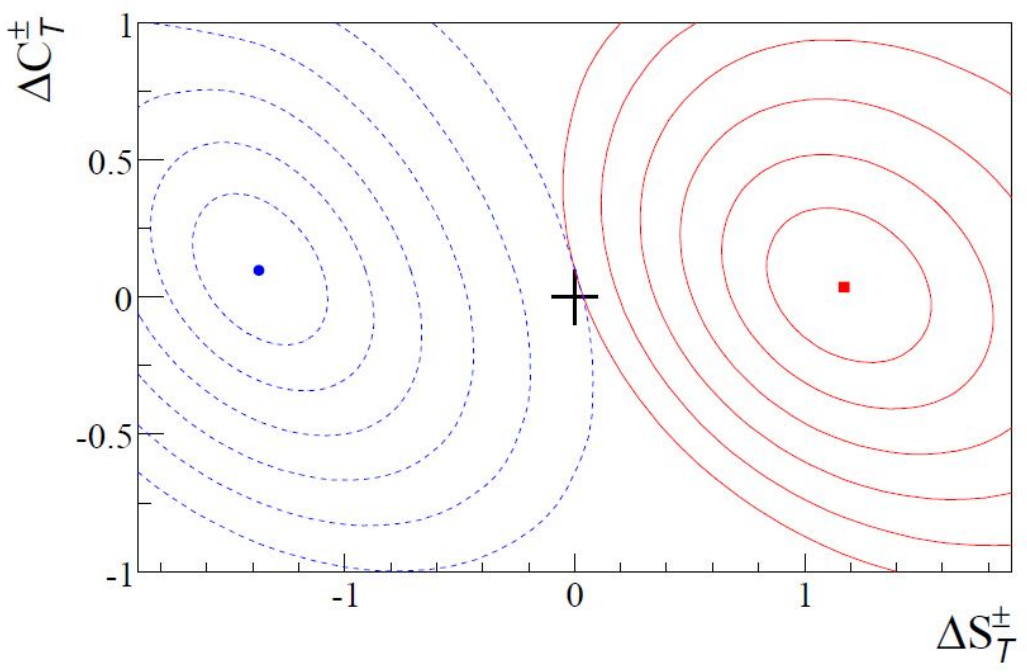

Figure 4: The values of the four $T$-violating asymmetries measured by BABAR, namely the differences in the two coefficients of the decay-time-dependent rates $S, C$ (the latter expected to be small in the SM) between $T$-transformed processes; the ${ }^{ \pm}$superscripts (+: blue, dashed, -: red, solid) indicate whether the decay to the flavour final state occurs before or after that of the companion meson to the CP final state. Ellipses correspond to 1 (one-dimensional) standard deviation intervals, with systematic uncertainties included. The cross indicates the $T$ symmetry point. From [108]. 
the above asymmetries exhibit $T$ violation in the interference of decays with and without mixing ( $T$ violation in decays cannot be directly tested, as it would require the genuine time reversal of a decay process).

\subsection{Electric dipole moments}

A different class of tests for $T$ symmetry violation avoids the above mentioned difficulties by considering a static process. Static Electric Dipole Moments (EDM) of (non-degenerate) elementary systems are odd under $P$ and $T$ transformations: this is in contrast to the usual electric dipole moments of polar molecules, for which a degeneracy is lifted by the orientation of the molecule in an external electric field, resulting in an interaction energy quadratic in the field magnitude ${ }^{6}$ Loosely speaking, this is because any such elementary EDM should be aligned with the spin (being the only preferred direction for a non-degenerate system), but spin and EDM have different transformation properties under $P$ and $T]^{7}$. Since $P$ violation in weak interactions is well established, EDM searches are usually considered as tests of $T$ symmetry. No spurious FSI effects are present in this case, since the initial and final states are the same, and a non-zero EDM would therefore be unambiguous proof of time symmetry violation.

The known $C P$ violation present in weak interactions induces unmeasurably small (but computable) effects, so that the search for EDMs is a field in which high precision is pursued. An additional reason for the interest in EDMs is related to the fact that they would most naturally arise from to non-flavour-changing interactions, such as the anomalous term in the QCD Lagrangian discussed in section 2.2. differently from the weak processes in which $C P$ violation is known to exist in the SM. A large background-free window of opportunity exists for detecting physics beyond the SM, which might in general contribute with large effects to EDMs.

Estimates for EDM magnitudes can be expressed as the product of the system size $L$ times the squared ratio of the Higgs vacuum expectation value $v$ to the mass scale $\Lambda$ associated to new particles, a dynamical factor $F$ involving to coupling

\footnotetext{
${ }^{6}$ Note also the analogies with $P$ and $T$ odd quantities appearing in solid-state physics, e.g. in magneto-electric crystals [111].

${ }^{7}$ There are ways of evading this conclusion, if other preferred directions do exist, such as in the case of Lorentz symmetry violation or non-commutativity of space-time coordinates, but these are arguably rather dramatic hypotheses.
} 
constants $g$ and loop factors, and the sine of a $C P$-violating phase $\phi$, i.e.

$$
d \sim L\left(\frac{v}{\Lambda}\right)^{2} F \sin \phi
$$

Experimental limits on EDMs thus constrain a combination of mass scale, coupling constant and $C P$-violating phases.

EDM searches are a mature sub-field with a 70-year long history, several dedicated reviews are available [112] [113], and only a summary outlook is possible here.

Most experiments look for a frequency (energy) shift of magnetic sub-levels in a spin-polarized neutral system immersed in an electric field: a difference in such frequency for opposite orientations of the electric field is the indication of the presence of an EDM; the required frequency resolution can be $O(\mathrm{nHz})$. The measurements require strong electric fields and well-known magnetic fields; the errors depend on the number of available particles and the measurement time, but also on a number of experimental effects, among which the shielding and measurement of magnetic fields are crucial. Furthermore, depending on the system, the extraction of the EDM from the measurement might require important theoretical input.

EDMs have been searched for in many systems, all measurements being so far consistent with zero. Different systems, besides the diversity in technique and experimental issues, are sensitive to different elementary EDMs and possible sources of $T$ violation, and therefore somewhat complementary to each other.

For neutral systems with a non-zero magnetic moment the measurement is conceptually simple, and indeed the neutron was the first system being probed [114]. The contribution to the neutron EDM by SM $C P$ violation is predicted to be $O\left(10^{-32} e \mathrm{~cm}\right)$ from loop effects, leaving ample ground for experimental tests of alternative sources of $T$ violation, such as the QCD $\theta$ term (section 2.2). Current limits are obtained with Ultra Cold Neutrons (UCNs) which, having kinetic energies of a few hundred neV (at mK temperatures), can be stored in matter bottles or by magnetic and gravitational fields for long times. The best limit was obtained at the ILL of Grenoble: $\left|d_{n}\right|<3 \cdot 10^{-26} e \mathrm{~cm}$ (at 90\% confidence level) [115]. Limitations are generally related to the statistics and the observation time, and to the spurious magnetic fields, including those induced by the motion of the neutrons, which require careful control. Recent progress in the production of UCNs using superfluid helium, new UCN sources and experimental approaches point to a possible 1-2 orders of magnitude improvement of the limit. 
The study of spin rotation in polarized neutron diffraction on the intense electric field in a crystal is a completely different technique which could possibly provide competitive neutron EDM measurements in the future.

The measurement of EDMs for charged particles usually requires these to be embedded within a neutral system, such as an atom or a molecule, to avoid them accelerating away from the observation region; in some cases the local electric fields in such systems can be much larger (up to $10^{12} \mathrm{~V} / \mathrm{m}$ ) than those which can be produced in the laboratory $\left(O\left(10^{7} \mathrm{~V} / \mathrm{m}\right)\right.$, thus offering a big advantage for the measurement. While the electrostatic equilibrium of the overall neutral system would seem to enforce the vanishing of the electric field at the position of any charged particle ("Schiff theorem"), finite-size and relativistic corrections alter such conclusion [116]. This allows the measurement of charged particles EDMs within neutral atoms or molecules to be performed, and introduces a conversion factor between the measured effect and the elementary EDM, leading in some cases to large enhancement factors, while on the other hand requiring theoretical (molecular, atomic or nuclear) calculations, which contribute additional uncertainties. Besides elementary EDMs, any hypothetical $T$-violating part of the interaction among the constituent particles can also result in an effective EDM, similarly to what was discussed for parity in section 2; the possible contribution due to different sources of $T$ violation is taken into account in the most recent analyses [112].

The electron EDM is predicted to be extremely small in the $\mathrm{SM}, \mathrm{O}\left(10^{-38}\right)$ $e \mathrm{~cm}$, arising via four-loop quantum effects involving virtual quarks (which do exhibit $C P$ violation in the SM). Relativistic effects (the contraction of the EDM for a moving particle) in paramagnetic systems with unpaired electrons (non-zero spin) result in an effective enhancement of an electron EDM by a factor $\sim 10 Z^{3} \alpha^{2}$ ( $Z$ being the atomic number and $\alpha$ the fine structure constant), which is large in heavy atoms, despite their small atomic polarizability.

The polarization of heavy atoms can be much larger when these are at one end of a diatomic polar molecules, resulting in much stronger internal fields and therefore larger effects: the electron cloud is spontaneously polarized along the intermolecular axis, which can be easily oriented in an electric field, resulting in a first-order interaction with it. The presence of an intrinsic linear Stark effect in the molecule requires to probe a resonance related to the nuclear spin; moreover, the chemical reactivity of the molecules poses some experimental issues not present with atoms.

Thanks to the above enhancement factors, EDM limits for electrons in heavy atoms or polar molecules are often strong: the current best limit on the elec- 
tron EDM is $\left|d_{e}\right|<1.1 \cdot 10^{-29} e \mathrm{~cm}$ (at $90 \%$ confidence level), obtained by the ACME collaboration [117] using thorium monoxide (ThO) molecules; the best limit obtained using thallium atoms is two orders of magnitude larger [118]. Future prospects involve novel trapping and cooling methods to allow longer measurement times for alkali atoms, and the use of cold beams and improved traps for paramagnetic polar molecules.

Special ferroelectric and paramagnetic solid-state crystals are also considered as promising systems for electron EDM measurements [112].

Diamagnetic systems (zero spin) shield any nuclear EDM, and relativistic effects are not effective, due to the slow nuclear motion. In this case what inhibits complete shielding of the external electric field is the finite size of the nucleus; the so-called nuclear Schiff moment [116] is the lowest-order residual effect, related to a difference between the EDM and charge distributions, which can be probed by low-lying atomic or molecular electrons. The main contribution to the Schiff moment would arise from possible $P$ - and $T$-violating nuclear interactions, although the EDMs of nucleons can also contribute. Because of the relative size of the nucleus and the electron cloud, the external electric field is shielded by large factors in diamagnetic atoms, but again in a diatomic polar molecule the electron cloud can be strongly polarized by a relatively small external field, canceling the shielding and even resulting in an enhancement. Shielding effects also result in easier experimental conditions (room temperature storage, long coherence times, optical spin polarization). Among the few volatile and polarizable diamagnetic atoms, ${ }^{199} \mathrm{Hg}$ provides the most sensitive EDM measurement so far: $\left|d_{e}\right|<7.4 \cdot 10^{-30} e \mathrm{~cm}$ (at 95\% confidence level) [119], also yielding an indirect limit on the proton EDM: $\left|d_{p}\right|<2.1 \cdot 10^{-25} e \mathrm{~cm}$ [120] (as well as an indirect limit on $\left|d_{n}\right|$ slightly better than the direct measurement). Diamagnetic systems in which the Schiff moment is particularly enhanced by octupole deformations $\left({ }^{225} \mathrm{Ra},{ }^{221 / 223} \mathrm{Rn}\right)$ appear promising for future measurements, and experiments are underway on several systems.

A nuclear Magnetic Quadrupole Moment (MQM), possible for nuclear spin 1 or larger, is also $P$ - and $T$-violating, and not shielded as an EDM. A value $(1.9 \pm 8.3) \cdot 10^{-34} e \mathrm{~cm}^{2}$ was measured in cesium [121] as a byproduct of an EDM experiment. A non-zero MQM can lead to permanent EDMs in atoms and molecules by mixing opposite parity states, and again atomic/molecular calculations are required to establish the connection.

A different approach for measuring EDMs of charged particles is that of having them circulate in a storage ring, and measuring the additional torque on the particle spin related to a EDM interaction $\boldsymbol{d} \times \boldsymbol{E}$, with the rest-frame electric field 
$\boldsymbol{E}$ being partly due to the motional field $\gamma \boldsymbol{v} \times \boldsymbol{B}$, which can be much larger than an externally applied field [122]. Similarly to what happens due to the anomalous magnetic moment, the spin of a rotating particle injected with longitudinal polarization will evolve in time, developing a component transverse to its velocity, if a non-zero EDM is present in suitably chosen fields. In the case of the muon, the polarization direction can be determined by its decay asymmetry, and for nuclei by their spin-dependent elastic scattering. This technique allows direct measurements on charged systems, with a high number of particles and a large measurement time (intrinsically limited by the lifetime in the case of the muon); in most models effects are expected to be larger for heavier particles.

A limit on the muon EDM was obtained as a byproduct of the anomalous magnetic moment $(g-2)$ measurement at BNL: $\left|d_{\mu}\right|<1.8 \cdot 10^{-19} e \mathrm{~cm}$ (at $95 \%$ confidence level) [123], although the optimal experimental conditions to measure $g-2$ are different from those required for an EDM measurement. The presence of radial magnetic fields allows the $g-2$ spin precession to mimic an EDM: one way to avoid this is the use of a suitable electric field to cancel the effect, or of purely electric confinement; still, spurious magnetic fields could contribute, and the comparison of counter-propagating beams was proposed to disentangle an EDM from the magnetic effect.

A limit on the EDM of the $\Lambda$ hyperon was similarly obtained as a byproduct of the magnetic moment measurement at Fermilab, exploiting the spin precession of polarized $\Lambda$ in a magnetic field, as measured by the angular asymmetry of its hadronic decay: $\left|d_{\Lambda}\right|<1.5 \cdot 10^{-16} e \mathrm{~cm}$ (at 95\% confidence level) [124].

Similar measurements might be performed on systems with much longer lifetimes, such as meta-stable nuclei, with their $\beta$ decays used for polarization analysis, or even stable nuclei, whose polarization can be probed through the asymmetry in elastic scattering on targets.

Experiments have been proposed for measuring EDMs of muons at JPARC and PSI, and of the proton, deuteron and helium ion at COSY and in dedicated rings: several ideas have been proposed to avoid the effect of the magnetic moment anomaly, and the goals for sensitivity range from $O\left(10^{-24} e \mathrm{~cm}\right)$ for muons to $O\left(10^{-29} e \mathrm{~cm}\right)$ for stable nuclei. An extensive list of references for current and future EDM experiment can be found in [84].

It might be finally mentioned that even a limit for the (electron anti-)neutrino EDM was set by analyzing a reactor experiment, on the basis that it would cause an anomalous ionization in a detector [125]: $\left|d_{v} F\right|<2 \cdot 10^{-20} e \mathrm{~cm}$, where $F$ is an (unknown) form factor; if the neutrino is a Majorana particle its electric (and magnetic) dipole moments are identically zero (but not so the $T$-even, $P$-odd 
anapole moments).

\section{CPT}

While all three discrete symmetries $C, P, T$ are definitely violated in Nature, their combination $C P T$ seems to hold absolutely, and such symmetry appears to be very deeply rooted in our description of Nature, being valid in the SM and in all consistent (quantum field) theories under very general (and experimentally verified) assumptions, according to the $C P T$ theorem. Proofs of such theorem are based on principles such as Lorentz invariance, locality and positivity of the energy, although several subtleties exist [126], and the theoretical validity of $C P T$ symmetry in realistic theories has been questioned [127]. It has been suggested that the fundamental nature of $C P T$ symmetry might be linked to the equivalence of the $P T$ reflection of all space-time axes to a rotation, which holds true for an even number of space-time dimensions, with the addition of $C$ being related to the Feynman-Stueckelberg interpretation of antiparticles. It is fair to say, however, that a simple and intuitive understanding of such a fundamental symmetry is still lacking, and its general validity is of course an issue to be addressed experimentally.

It is generally held that a violation of Lorentz symmetry would entail the violation of $C P T$ and vice versa [128], even if this is now understood not to be absolutely true, and the validity of the above two symmetries might be independent in some models 8 [129]. We thus briefly mention that tests of Lorentz symmetry have been performed (with negative results) on many systems, e.g. by looking for a preferred direction in space, which would induce a dependence of physical observables on sidereal time. A comprehensive framework for analyzing limits on the effective violation of Lorentz symmetry induced by physics at very high mass scales was developed [130], but a discussion of such tests is outside the scope of this article.

So far, no hint of violation of $C P T$ symmetry was reported by any experiment, and some numerically impressive limits have been obtained.

\footnotetext{
${ }^{8}$ These are admittedly "exotic", involving non-commutative geometry of space-time or nonlocal interactions, but arguably, when considering such a wild thing as $C P T$ violation, nothing can be barred.
} 


\subsection{Anti-particle properties}

While, strictly speaking, there is no symmetry between particles and anti-particles due to the lack of exact $C$ symmetry, $C P T$ symmetry itself imposes several constraints ${ }^{9}$ on their properties, some of which have been tested experimentally.

$C P T$ symmetry implies the equality of the (absolute values of) masses, lifetimes, charges, magnetic moments, etc. for particles and anti-particles, and many such comparisons have been performed, with varying degrees of precision, with no difference being ever measured. The numerical value of some limit on a relative particle/anti-particle difference cannot be taken as a unique relevant figure of merit since in general, lacking a model for $C P T$ violation, effects in different systems cannot be compared. This should be kept in mind when discussing different results, and should also remind that tests on many different systems are valuable, irrespective of their relative precision. The most precise direct comparisons are performed on stable particles.

The spectroscopic measurement of the energy difference between the $2^{3} S_{1}$ and $1^{3} S_{1}$ levels of positronium, using counter-propagating laser beams to reduce Doppler broadening, allowed to reach a precision of $\sim 2.5$ parts per billion [132], and the agreement with the theoretical calculations constrains the electronpositron mass difference to be $<8 \cdot 10^{-9} \mathrm{~m}_{e}$ (at $90 \%$ confidence level).

An intense program for testing $C P T$ exploiting anti-protons is being pursued at the CERN Anti-proton Decelerator, and the capability of forming and manipulating anti-hydrogen $\left(\bar{p} e^{+}\right)$[133] opened many interesting opportunities.

The lifetime of anti-protonic helium $\left(\bar{p} \mathrm{He}^{+}\right)$, in which an anti-proton replaces one electron in a highly excited level, is large enough to allow laser spectroscopy: a measurement of $\bar{p}$ transition frequencies to a precision of few parts per billion [134], and the comparison with theory, allowed the ASACUSA experiment to constrain the proton/anti-proton mass and charge magnitude relative differences to $7 \cdot 10^{-10}$ (at $90 \%$ confidence level). The $1 \mathrm{~S}-2 \mathrm{~S}$ transition energy in anti-hydrogen is now measured to match that of hydrogen at a precision $\sim 2 \cdot 10^{-12}$ by the ALPHA experiment [135], with a steady improvement in the control of systematic effects, to which long (days) trapping times of individual anti-atoms contribute. Such results actually used the more precise information on the equality of the proton and anti-proton charge-to-mass ratios, measured to a precision of $9 \cdot 10^{-11}$ by the CERN TRAP collaboration [136] by comparing the cyclotron frequencies of a $\bar{p}$ and a $H^{-}$ion simultaneously trapped in a Penning trap; such result has since been improved since by the BASE measurement with a precision of $6.9 \cdot 10^{-11}$ [137].

\footnotetext{
${ }^{9}$ Some of these constraints may survive even in presence of $C P T$ violation, see e.g. [131].
} 
The comparison of magnetic moments deserves a special mention due to the remarkable relative precision which can be obtained through frequency measurements: it is now $2.1 \cdot 10^{-12}$ for the $e^{+} e^{-}$comparison [138], obtained by comparing the spin-cyclotron difference frequency to the cyclotron frequency for single electrons and positrons in a Penning trap (so-called "geonium" atoms), and $8 \cdot 10^{-7}$ for the $\bar{p} p$ comparison [139].

The masses and lifetimes of long-lived baryons, mesons and hyperons were also compared to those of the respective anti-particles, with varying levels of precision $O\left(10^{-3} \div 10^{-5}\right)$ [35]. The neutral kaon system stands out in this respect, and is discussed in the following section.

The range of $C P T$ tests based on particle/anti-particle mass differences extends to the heaviest known elementary particle, the comparison of top and antitop quark masses being $\left(m_{t}-m_{\bar{t}}\right) / m_{t}=(-0.9 \pm 1.1) \cdot 10^{-3}$, dominated by the CMS measurement [140]. Tests on nuclear anti-matter have been performed as well, at the heavy ion colliders RHIC and LHC: the interaction between anti-protons was shown to be attractive and consistent with that of protons [141], the chargeto-mass ratio for anti-deuterons and anti-helium-3 were measured to be equal to those of their matter counterparts to $10^{-4}$ [142], the mass difference between the hypertriton ${ }_{\Lambda}^{3} \mathrm{H}$ ( $p n \Lambda$ bound system) and its anti-matter counterpart was measured to be consistent with zero to $\sim 10^{-4}[143]$, thus probing the $C P T$ symmetry also for strange quark nuclear binding.

It should be noted that $C P T$ symmetry imposes the equality of inertial masses for particles and anti-particles, but has nothing to say about their gravitational masses, although the (weak) equivalence principle states that these two masses do coincide. While several arguments have been put forward against the possibility that anti-matter gravitates differently from matter, direct tests of the equality of free fall for particles and anti-particles are lacking, and actively pursued. The gravitational force on an electron on earth can be balanced by an electric field of magnitude just $\sim 6 \cdot 10^{-11} \mathrm{~V} / \mathrm{m}$; even if macroscopic fields were controlled to such formidable level in a region shielded by conducting material, electric fields due to microscopic non-uniformities are believed to represent an uneliminable limit, and experiments are mostly performed on neutral systems such as anti-hydrogen [144] (although limits on the gravitational-inertial mass difference to $0.13 \%$ were set for relativistic $e^{+}$circulating in a particle accelerator, thanks to the absence of anomalous radiative energy losses [145]). So far the best limit indicates that anti-hydrogen does not fall up with a gravitational mass more than 65 times larger than its inertial mass (at 95\% confidence level) [146]; results are expected to reach the interesting range to experimentally test the anti-gravity hypothesis in the next 
years.

Stringent limits were also obtained by the absence of measured effects related to the time variation of the gravitational potential on the highly sensitive $K^{0} \bar{K}^{0}$ system, ranging from $O\left(10^{-4}\right)$ to $O\left(10^{-14}\right)$ depending on the type and range of the assumed interaction [147].

We finally note that $C P T$ symmetry imposes not only the equality of total decay rates, but also that for partial decay rates into sets of states not connected by re-scattering effects, since any rate difference in such modes cannot be "compensated" by other modes; this can be exploited for kaons, thanks to the small number of available decay modes, and indeed the equalities of $K^{ \pm} \rightarrow \mu^{ \pm} v$ and $K^{ \pm} \rightarrow \pi^{ \pm} \pi^{0}$ decay rates provide tests of $C P T$ symmetry at the $10^{-3}$ level.

\subsection{Indirect tests}

If $C P T$ symmetry holds in the $K^{0}-\bar{K}^{0}$ mixing process (and therefore the $C P$ violation of such process also exhibits a corresponding $T$ violation), the phase of the $\epsilon$ parameter quantifying the $C P$ impurity of the physical states is constrained by the mass and lifetime differences of the states to be close to $\arctan (-2 \Delta m / \Delta \Gamma)=$ $(43.52 \pm 0.05)^{\circ}$. Conversely, if $T$ symmetry holds and $C P$ violation is accompanied by $C P T$ violation, such phase would be shifted by $90^{\circ}$ from the above value (see e.g. [2]). The phase can be measured by studying the time dependence of the $\pi \pi$ decay rate starting from flavour eigenstates, and its value [35] is $(43.5 \pm 0.5)^{\circ}$, fully consistent with CPT symmetry.

The same measurements allow to constrain the presence of $C P T$ violation in $K \rightarrow \pi \pi$ decays, through the difference in the phases of the $C P$-violating amplitude ratios for the $\pi^{0} \pi^{0}$ and $\pi^{+} \pi^{-}$channels, which is measured to be $(0.34 \pm 0.32)^{\circ}$ [35], fully consistent with the close-to-zero value $(0.006 \pm 0.008)^{\circ}$ expected in case of $C P T$ symmetry (such phase difference is also expressed as the imaginary part of the ratio of direct to indirect $C P$-violating parameters $\mathfrak{J}\left(\epsilon^{\prime} / \epsilon\right)$ ).

Semi-leptonic $K_{L}$ decays are similarly measured to respect $C P T$ symmetry to $O\left(10^{-3}\right)$, and a comparison of the $C P$-violating charge asymmetry with that measured in $K_{S}$ (so far compatible with zero within errors) provides a test of $C P T$ at a precision of few $10^{-3}$ [148], expected to slightly improve with the full analysis of KLOE-2 data. A related clean test of $C P T$ symmetry is obtained by the comparison of transition rates between flavour and $C P$ eigenstates [149], not yet measured but accessible to KLOE-2, which might reach $\sim 10^{-3}$ precision on it. 
The small number of decay modes available to kaons allows the use of unitarity ("Bell-Steinberger") relations to put limits on $C P$ and $C P T$ violating terms from the knowledge of the decay widths of the physical states $K_{S}$ and $K_{L}$. A $C P T$-violating parameter $\delta 10$ in $K^{0}-\bar{K}^{0}$ mixing is constrained by measurements and unitarity to be $\delta=[(2.4 \pm 2.3)+i(-0.7 \pm 1.4)] \cdot 10^{-5}[35]$, consistent with zero. A non-zero $\delta$ would induce a mass and width difference between $K^{0}$ and $\bar{K}^{0}$, and the smallness of the mass difference between the physical states $\left(m_{K_{L}}-m_{K_{S}} \simeq\right.$ $3.5 \mu \mathrm{eV}$ ) turns this into impressive limits on such $C P T$-violating differences: assuming no $C P T$ violation in the decays of neutral kaons (i.e. $\Gamma_{K^{0}}=\Gamma_{\bar{K}^{0}}$ ) one gets [35]

$$
\left|m_{K^{0}}-m_{\bar{K}^{0}}\right| / m_{K^{0}}<6 \cdot 10^{-19}
$$

(at $90 \%$ confidence level), which is by far the most precise test of $C P T$.

Further indirect limits on the violation of $C P T$ symmetry can be set by considering that differences between charged particles and their anti-particles would induce other measurable effects: mass differences would result in a non-zero photon mass [150], so that the stringent limits on such quantity can be translated into order of magnitude limits on $e . g$. the $e^{+} e^{-}$mass difference, of (relative) order $10^{-23}$, much stronger than direct limits. Similarly, any difference in the magnitude of the charge for $e^{+}$and $e^{-}$would result in the non-neutrality of matter due to the polarization of the vacuum in the electric field inside atoms: the limit on the neutrality of atoms was translated into a limit $\left|Q\left(e^{+}\right)+Q\left(e^{-}\right)\right| /|e|<2 \cdot 10^{-18}[151]$, ten orders of magnitude stronger than the direct limits obtained by comparing the $e^{+}$and $e^{-}$cyclotron frequencies.

\subsection{Tests with neutrinos}

Any difference in masses or mixing angles between neutrinos and anti-neutrinos would be a sign of $C P T$ violation, which was proposed (and later dismissed) as a possible interpretation of inconsistencies among neutrino experiments [152]. In the analysis of oscillations, spurious environmental $C P T$ asymmetries due to the propagation through matter must be taken into account, although in the experiments discussed below such effects are quite negligible.

By comparing the squared mass difference for MeV-energy solar and reactor neutrino data (sources of $v_{e}$ and $\bar{v}_{e}$, respectively), interpreted in terms of twoflavour oscillations, the limit $\left|\Delta m_{12}^{2}(v)-\Delta m_{12}^{2}(\bar{v})\right|<1.1 \cdot 10^{-4} \mathrm{eV}^{2}$ was obtained [153], dominated by the uncertainty in the solar neutrino data.

\footnotetext{
${ }^{10}$ No relation to the phase in the CKM matrix for which the same symbol is used.
} 
The MINOS collaboration reported a combined study of $v_{\mu}, \bar{v}_{\mu}$ disappearance for $\mathrm{GeV}$-energy accelerator neutrinos and $O\left(1-10^{4} \mathrm{GeV}\right)$ atmospheric neutrinos, exploiting the magnetic detector which can distinguish $v$ and $\bar{v}$ on an event-byevent basis. From the analysis of the full data set of the experiment $\left(1.4 \cdot 10^{21}\right.$ POT, $735 \mathrm{~km}$ away, and $38 \mathrm{kton}$ year mass exposure) [154] $\Delta m_{32}^{2}(v)-\Delta m_{32}^{2}(\bar{v})=$ $\left(-1.2_{-2.6}^{+2.4}\right) \cdot 10^{-4} \mathrm{eV}^{2}$ is quoted, with consistency between accelerator and atmospheric data.

The T2K experiment also reported [155] results for accelerator neutrinos and anti-neutrinos squared mass differences consistent with the above. While atmospheric neutrinos and anti-neutrinos cannot be individually distinguished by Super-Kamiokande, their differences in flux and interaction cross section allow extracting information on $C P T$ symmetry from the $v_{\mu}, \bar{v}_{\mu}$ disappearance probability distributions as a function of zenith angle; the analysis of about half the currently available data was found to be fully consistent with $C P T$ symmetry [156].

In general, $C P T$ limits on the differences of neutrino and anti-neutrino mixing angles are numerically weak compared to those on squared mass differences, e.g. $\sin ^{2}(2 \theta)_{v}-\sin ^{2}(2 \theta)_{\bar{v}}=0.02 \pm 0.09$ is reported by MINOS [154].

It is worth noting that differences between the gravitational behavior of neutrino and anti-neutrino were constrained to be below $10^{-6}$ by the observed arrival time dispersion $O(12 \mathrm{~s})$ of the $19 v_{e}$ and $\bar{v}_{e}$ interaction events detected after the collapse of supernova SN 1987A [157].

\section{The universe at large}

When dealing with very large scales, gravity cannot be ignored any longer, and rather becomes the dominant interaction, in a way which we have very little experience of, and which we cannot study using the (Galilean) experimental method, since we cannot perform controlled experiments. Considering moreover the fact that we currently lack any microscopic description of gravity, it should be clear that the study of discrete symmetries in such an environment is at best shaky ground (see e.g. [158]). After such a mandatory disclaimer, we can state with some light-heartedness that deep connections between discrete symmetries and our description of the evolution of the universe are known, and very briefly mention some cosmological observations related to discrete symmetries. 


\subsection{Parity in space}

A peculiar observation is that the handedness of spiral galaxies appears to be nonuniformly distributed, i.e. the number of galaxies observed to be rotating clockwise or anti-clockwise is different in a statistically significant way [159]. An automated analysis of more than $10^{5}$ spiral galaxies, performed with attention to avoiding possible biases, shows that the local universe (redshift $z<0.3$ ) does not appear to be isotropic in terms of spiral galaxy handedness, with asymmetries depending on the right ascension coordinate, and a probability of occurring by chance of $\sim 6 \cdot 10^{-6}$ [160]; the distribution is consistent with the presence of a dipole axis direction, suggesting an apparent large-scale violation of parity.

\subsection{Charge conjugation in space}

It is a fact that anti-matter is not found in the visible universe, except for that which is produced in high-energy collisions between matter particles [161], [162]. A long-standing experimental program is being pursued to search for primordial anti-particles; while anti-protons and positrons are easily produced in high-energy interactions of matter, and hints of some of those being of primary origin require the assessment of excesses with respect to predictive models [163], the detection of heavier anti-nuclei would most likely indicate primordial anti-matter (or dark matter production mechanisms). Anti-matter nuclei up to anti-helium-4 have been produced in the laboratory using heavy ion collisions [164]; the measured exponential decrease of the anti-nuclei production yields with increasing number of anti-nucleons, $O\left(10^{-3}\right)$ per anti-nucleon, consistent with thermodynamic expectations, indicates that anti-lithium- 6 is out of reach of present accelerator technology.

The AMS-02 detector on the International Space Station might have detected a handful of anti-helium events [165], but with a signal to background $O\left(10^{-9}\right)$ any claim requires a thorough study of instrumental effects, that the collaboration is pursuing.

Indirect constraints based on the intensity of the cosmic diffuse $\gamma$-ray background put limits on the size of a possible domain structure of the universe in which some regions are dominated by matter and others by anti-matter: the minimum size of such domains, should they exist, is comparable to that of the observable universe [166].

The universe is thus very strongly dominated by matter, with the value of the Baryon Asymmetry of the Universe (BAU) $\eta=\left(n_{B}-n_{\bar{B}}\right) /\left(n_{B}+n_{\bar{B}}\right)\left(n_{B}, n_{\bar{B}}\right.$ being 
the densities of baryons and anti-baryons) determined to be $\sim 10^{-10}$, both by the abundance of light elements in the intergalactic medium and the power spectrum of the temperature fluctuations of the Cosmic Microwave Background (CMB). A large lepton asymmetry might be hidden in the cosmic neutrino backgrounds, but information is very rough in this case, and asymmetries up to $50 \%$ cannot be excluded.

The present state of the universe appears to be dramatically charge asymmetric.

\subsection{CP and baryogenesis}

The idea that a BAU is built in our universe as an asymmetric initial condition might not be aesthetically appealing: while this is not a scientific criterion for dismissing it, nowadays a more solid one is commonly accepted, namely the fact that inflation would wash out any initial net baryon number (although this is not a completely closed issue, see e.g. [167]). If such widely accepted scenario is true, the observed BAU must then arise dynamically during the evolution of the universe, starting from an effective zero net baryon number.

The seminal paper by Sakharov [168] elucidated the necessary conditions which must be simultaneously satisfied for this to happen, namely (i) baryon number non-conservation, (ii) $\mathrm{C}$ and $\mathrm{CP}$ violation, and (iii) departure from thermal equilibrium. The need for the above conditions can be readily understood in that (i) is clearly needed to change the net baryon number, (ii) is required to avoid that baryons and anti-baryons are created in the same amounts, and (iii) to ensure that the inverse processes do not cancel any generated baryon asymmetry. While it is true that baryogenesis models have being conceived in which any one of the Sakharov conditions can be dispensed for, CP violation remains a "natural" ingredient in almost all viable mechanisms for generating a $\mathrm{BAU}^{11}$.

The Standard Model might arguably include all three Sakharov ingredients. While baryon number (just as lepton flavour number) is accidentally conserved and cannot be violated perturbatively in the SM, non-perturbative (sphaleroninduced) reactions [169] can become relevant at high energies (temperatures) and induce effective baryon number non-conservation (while conserving the difference of baryon and lepton numbers). Non-equilibrium might be provided in the

\footnotetext{
${ }^{11}$ A statement whose relevance can be questionable, since assigning "probabilities" to cosmological models is at best a shaky concept, if not complete nonsense. But let's pretend to be pragmatic working physicists.
} 
SM through a strong-enough electroweak phase transition (occurring when the temperature of the universe was $\mathrm{O}(150 \mathrm{GeV})$, i.e. $\sim 10^{-12} \mathrm{~s}$ after the Big Bang), and of course weak interactions include $\mathrm{C}$ and $\mathrm{CP}$ violation. It is generally agreed, however, that the tiny magnitude of CP violation in the SM (as expressed by the measure $J$, see section 4) and the strength of the electroweak phase transition are not sufficient [170] to explain the measured BAU using the above ingredients ${ }^{12}$ In this sense one might say that our own existence is indeed the first evidence for physics beyond the SM (and a $C P$-violating one, for that!).

As noted above, physicists are clever at discovering theorems but also at finding counterexamples, so some remarks are due on the loopholes of the above statements. The requirement of thermal non-equilibrium for dynamical BAU generation is actually related to CPT symmetry [171]. It was noted [172] however that, without introducing any explicit violation of such a "sacred" symmetry, a slowlyvarying cosmological field (e.g. related to dark energy or to curvature) introduces an alternative "arrow of time" with respect to the thermodynamic one. This represents an effective spontaneous violation of Lorentz invariance, and therefore of CPT symmetry, thus allowing BAU generation even in thermal equilibrium ("spontaneous baryogenesis"). It was also speculated that the $C P T$ symmetry of the expanding universe as a whole could be recovered if before the Big Bang the universe was the $C P T$-symmetric version of our own, which can be interpreted as a universe/anti-universe pair emerging from nothing [173].

An important class of models for the generation of the BAU is the one which relies on the presence of new heavy fermions or scalars, often introduced to explain the existence of non-zero (but small) neutrino masses (a solid indication of physics beyond the SM). The lepton-number violating (and $C P$-violating) interactions of such particles, whose decays get out of equilibrium when the temperature of the universe drops below their mass, naturally generate a leptonic asymmetry; such leptogenesis [174] then generates a BAU through the non-perturbative SM reactions mentioned above, which convert the leptonic asymmetry into a baryonic asymmetry.

\subsection{CPT in space}

The expanding universe is intrinsically time asymmetric, arguably providing a natural frame in which Lorentz and $C P T$ symmetries might be broken. Speculations about a possible $C P T$ violation at the cosmological scale have been put forward,

\footnotetext{
${ }^{12}$ Although this one too is not an inescapable conclusion in all models.
} 
e.g. concerning the new physics which should be responsible for the assumed mechanism of inflation. One possible consequence of such violation is cosmological birefringence [175], i.e. the rotation of the polarization plane of electromagnetic waves during their propagation, due to the different velocity of circularly polarized modes of opposite handedness. Such an effect might be observable by considering radiation from distant radio-galaxies, which often exhibit an extended spatial structure correlating to the polarization of the emitted radiation. Another signature for such effects arises from the study of the CMB polarization. This can be decomposed in terms of two types of modes: the parity-even " $E$ " modes and the (so far undetected) parity-odd "B" modes; any cross-correlation between the two, or between a "B" mode and the CMB temperature, would be a signature of parity violation [176]. It was remarked that, while the above effects are usually considered as constraining $C P T$ symmetry, the possibility that they arise from $C P T$-conserving ( $P, C$-violating) interactions is not excluded [177]

No significant indications for such effects have been obtained so far by groundbased, balloon or satellite experiments, but this is a very active field of study (see e.g. [178]).

\section{Coda}

The emergence of symmetry as a guiding principle for understanding Nature is a conquest of reason, which allowed us to see beyond the apparent disorder and gross asymmetries of the world around us. The realization that many such symmetries are actually only imperfect ones was instead forced upon us by the deeper and precise experimental investigation of minute effects and processes, with little or no speculative input. This state of affairs turned out to be extremely fruitful in shaping the paradigm through which we currently (believe we can) interpret the physical world, as well as in bringing up ideas on what we know that we do not know.

The experimental program of searching for effects violating the approximate symmetries of Nature has been going on for more than sixty years, with ever growing momentum, and shows no sign of slowing down: an indication of the fact that it is a promising approach to gather information about the way our universe works. This appears to be particularly true in present times, when the direct discovery of new fundamental constituents of the world is slow to come, and conversely theoretical speculations are wild, due to the vast range of possibilities. More and more different systems are being investigated with increasing ingenuity 
and precision, and new facilities and experiments are being deployed, which have as central goals the study of the asymmetries of Nature.

If the twentieth century had many reasons to be deemed "the century of symmetries" [179], from such perspective it seems to us that the progress of physics in the twenty-first will still be strongly shaped by the investigation of such concept. If this is true, we can be assured that experimental results will be the keystones for securing such process.

\section{References}

[1] Particle Data Group: PDGLive at http://pdglive.lbl.gov.

[2] Sozzi M S 2008 Discrete Symmetries and CP Violation (Oxford: Oxford University Press).

[3] Wu C S et al. 1957 Phys. Rev. 105 1413; Garwin R L et al. 1957 Phys. Rev. 105 1415; Friedman J I, Telegdi V L 1957 Phys. Rev. 1051681.

[4] Akimov D et al. 2017 (COHERENT collaboration) Science 3571123.

[5] Erler J et al. 2014 Ann. Rev. Nucl. Part. Sci. 64269.

[6] Roberts B M et al. 2015 Ann. Rev. Nucl. Part. Sci. 6563.

[7] Wood CS et al. 1997 Science 2751759.

[8] Antypas D et al. 2019 Nat. Phys. 15120.

[9] Zel'dovich Ia B 1957 J. Exptl. Theoret. Phys. (USSR) 331531.

[10] Haxton W C, Wieman C E 2001 Ann. Rev. Nucl. Part. Sci 51261.

[11] Kharzeev D, Pisarski R D 2000 Phys. Rev. D 61 111901(R).

[12] Adamczyk L et al. (STAR collaboration) 2013 Phys. Rev. C 88064911.

[13] Adam J et al. (ALICE collaboration) 2016 Phys. Rev. C 93044903.

[14] Khriplovich I B, Rudenko A S 2012 Phys. Atom. Nucl. 751142.

[15] Ramsey-Musolf M J, Page S A 2006 Ann. Rev. Nucl. Part. Sci. 561. 
[16] Cheng H-Y 1988 Phys. Rep. 158 1; Kim J E, Carosi G 2010 Rev. Mod. Phys. 82557.

[17] Graham P W, Rajendram S 2011 Phys. Rev. D 84055013.

[18] Macq P C et al. 1958 Phys. Rev. 1122061.

[19] Ambrosino F et al. (KLOE collaboration) 2008 J. High Energ. Phys. 05 006.

[20] McDonough J et al. (Crystal Box collaboration) 1988 Phys. Rev. D 382121.

[21] Mills A P, Berko S 1967 Phys. Rev. Lett. 18420.

[22] Vetter P A, Freedman J 2002 Phys. Rev. A 66052505.

[23] Bass S D 2019 Acta Phys. Pol. B 501319.

[24] Frére J-M 2012 Compt. Rend. Phys. 13104.

[25] Landau L 1957 Soviet Phys. JETP 5336.

[26] Branco G C, Lavoura L, Silva J P 1999 CP violation (Oxford: Oxford University Press).

[27] Bigi I, Sanda A I 2009 CP violation, 2nd edition (Cambridge: Cambridge University Press).

[28] Kleinknecht K 2003 Uncovering CP violation (Berlin, Heidelberg: Springer Verlag).

[29] Chen M-C et al. 2014 Nucl. Phys. B 883267.

[30] Christenson J H et al. 1964 Phys. Rev. Lett. 13138.

[31] Kobayashi M, Maskawa T 1973 Prog. Theor. Phys 49652.

[32] Wolfenstein L 1964 Phys. Rev. Lett. 13562.

[33] Alavi-Harati A et al. (KTeV collaboration) 1999 Phys. Rev. Lett. 83 22; Fanti V et al. (NA48 collaboration) 1999 Phys. Lett. B 465 335; Aubert B et al. (BABAR collaboration) 2001 Phys. Rev. Lett. 86 2515; Abashian A et al. (Belle collaboration) 2001 Phys. Rev. Lett. 862509. 
[34] Jarlskog C 1985 Phys. Rev. Lett. 551039.

[35] Tanabashi M et al. (Particle Data Group) 2018 Phys. Rev. D 98030001.

[36] Charles J et al. (CKMfitter group) 2005 Eur. Phys. J. C 41 1; updated results available at http: //ckmfitter.in2p3.fr.

[37] Bona M et al. (UTfit group) 2006 J. High Energ. Phys. 10 081; updated results available at http://wWw.utfit.org.

[38] Berger J et al. 2012 J. High Energ. Phys. 10181.

[39] Khachatryan V et al. (CMS collaboration) 2017 J. High Energ. Phys. 2017 101; Aaboud M et al. (ATLAS collaboration) 2017 J. High Energ. Phys. 201771.

[40] Alavi-Harati A et al. (KTeV Collaboration) 2002 Phys. Rev. Lett. 88 181601.

[41] Angelopoulos A et al. (CPLEAR collaboration) 2003 Phys. Rep. 374165.

[42] Franzini P, Moulson M 2006 Ann. Rev. Nucl. Part. Sci. 56 207; AmelinoCamelia G et al. (KLOE-2 collaboration) 2010 Eur. Phys. J. C 68619.

[43] Batley J R et al. (NA48 collaboration) 2002 Phys. Lett. B 544 97; Abouzaid E et al. (KTeV collaboration) 2011 Phys. Rev. D 83 092001; Sozzi M S 2004 Eur. Phys. J. C 3637.

[44] Bailey J A et al. (SWME collaboration) 2018 Phys. Rev. D 98094505.

[45] Bai Z et al. (RBC and UKQCD collaborations) 2015 Phys. Rev. Lett. 115 212001.

[46] Buras A 2018 Acta Phys. Pol. B 491043.

[47] Babusci D et al. (KLOE-2 collaboration) 2013 Phys. Lett. B 72354.

[48] Batley J R et al. (NA48/2 collaboration) 2007 Eur. Phys. J. C 52875.

[49] Cortina Gil E et al. (NA62 collaboration) 2019 Phys. Lett. B 791; Ahn J K et al. (KOTO collaboration) 2019 Phys. Rev. Lett. 122021802. 
[50] Aubert B et al. (BABAR Collaboration) 2001 Phys. Rev. Lett. 87 091801; Abe K et al. (Belle Collaboration) 2001 Phys. Rev. Lett. 87091802.

[51] Bevan A J et al. (eds.) 2014 Eur. Phys. J. C 743026.

[52] Lewis J, Van Kooten R 2015 Int. J. Mod. Phys. A 301541003.

[53] Adeva B (LHCb collaboration) 2016 EPJ Web of Conferences 12602001.

[54] Lenz A et al. (CKMfitter group) 2011 Phys. Rev. D 83036004.

[55] Amhis Y et al. (HFLAV, Heavy Flavour Averaging Group, formerly HFAG) 2017 Eur. Phys. J. C 77 895, latest results available at https://hflav. web.cern.ch/.

[56] Abazov V M et al. (D0 collaboration) 2014 Phys. Rev. D 89012002.

[57] Aaij R et al. (LHCb collaboration) 2016 Phys. Rev. Lett. 117 061803; Aaij R et al. (LHCb collaboration) 2017 Phys. Rev. Lett. 118129903.

[58] Abe K et al. (Belle collaboration) 2004 Phys. Rev. Lett. 93021601.

[59] Aaij A et al. (LHCb collaboration) 2012 Phys. Rev. Lett. 108201601.

[60] Aaij A et al. (LHCb collaboration) 2014 Phys. Rev. D 90112004.

[61] Aaij R et al. (LHCb collaboration) 2019 report CERN-EP-2019-062, preprint arXiv: 1905.09244.

[62] Bediaga I et al. (LHCb collaboration) 2018 Physics case for an LHCb Upgrade II, report CERN-LHCb-PUB-2018-009, preprint arXiv: 1808.08865 .

[63] Kou E et al. (Belle-II collaboration) 2018 The Belle II physics book, KEK preprint 2018-27, preprint arXiv: 1808.10567.

[64] Aaij A et al. (LHCb collaboration) 2019 Phys. Rev. Lett. 122 211803, preprint arXiv: 1903.08726v2.

[65] Pontecorvo B 1957 J. Exptl. Theoret. Phys. 33549 [Sov. Phys. JETP 7 (1958) 429.]; Maki Z, Nakagava M, Sakata S 1962 Prog. Theor. Phys. 28 870. 
[66] Wolfenstein L 1978 Phys. Rev. D 172369.

[67] Johnson M B et al. 2015 Phys. Rev. D 91076005.

[68] Bernabéu J, Segarra A 2019 J. High Energ. Phys. 03103.

[69] Abe K et al. (T2K collaboration) 2018 Phys. Rev. Lett. 121171802.

[70] Acero M A et al. (NOvA collaboration) 2018 Phys. Rev. D 98032012.

[71] Abe K et al. (Super-Kamiokande collaboration) 2018 Phys. Rev. D 97 072001.

[72] Acciarri R et al. (DUNE collaboration) 2016 Long-Baseline Neutrino Facility (LBNF) and Deep Underground Neutrino Experiment (DUNE) Conceptual Design Report.

[73] Abe K et al. (Hyper-Kamiokande collaboration) 2016, Hyper-Kamiokande Design Report, KEK Preprint 2016-21.

[74] Inami K et al. (Belle collaboration) 2003 Phys. Lett. B 55116.

[75] Branco G C et al. 2012 Rev. Mod. Phys. 84515.

[76] Arntzenius F, Greaves H 2009 Brit. J. Phil. Sci. 60557.

[77] Roberts B W 2017 Philos. Sci. 84315.

[78] Bernabéu J, Martínez-Vidal F 2015 Ann. Rev. Nucl. Part. Sci. 65403.

[79] Lindroos M, Mezzetto M 2010 Ann. Rev. Nucl. Part. Sci. 60299.

[80] Henley E M et al. 2011 Int. J. Mod. Phys. E 20 2463, and 2012 Int. J. Mod. Phys. E 211292001.

[81] Sobków W, Blaut A 2018 Eur. Phys. J C 78197.

[82] Wolfenstein L 1999 Phys. Rev. Lett. 83911.

[83] Herczeg P, Khriplovich I B 1997 Phys. Rev. D 5680.

[84] Chupp T E et al. (emiT-II collaboration) 2012 Phys. Rev. C 86035505.

[85] Kozela A et al. (nTRV collaboration) 2012 Phys. Rev. C 85045501. 
[86] Abe M et al. (E246 collaboration) 2004 Phys. Rev. Lett. 93131601.

[87] Paton K et al. 2006 Measurement of T-violating Transverse Muon Polarization in $K^{+} \rightarrow \pi^{0} \mu^{+} v$ Decays, J-PARC experimental proposal.

[88] Dannenberg M et al. 2005 Phys. Rev. Lett. 94021802.

[89] Yamazaki T et al. 2010 Phys. Rev. Lett. 104083401.

[90] Vetter P A, Freedman S J 2003 Phys. Rev. Lett. 91263401.

[91] Gajos A et al. (J-PET collaboration) 2018 Adv. High En. Phys. 2018 8271280 .

[92] Braguta V V et al. 2002 Phys. Rev. D 65054038.

[93] Khriplovich I B, Rudenko A S 2011 Phys. Atom. Nucl. 741214.

[94] Akimenko S A et al. (ISTRA+ collaboration) 2007 Phys. Atom. Nucl. 70 702.

[95] Bensalem W, London D 2001 Phys. Rev. D 64116003.

[96] del Amo Sanchez P et al. (BABAR collaboration) 2010 Phys. Rev. D 81 111103(R); Lees J P et al. (BABAR collaboration) 2011 Phys. Rev. D 84 031103(R).

[97] Gardner S, He V 2012 Phys. Rev. D 86016003.

[98] Gao D-N 2002 Mod. Phys. Lett. A 17 1583, and references therein.

[99] Ambrosino F et al. 2009 (KLOE collaboration) Phys. Lett. B 675283.

[100] Valencia G 2012 Proc. of Science HQL2012 050.

[101] Kabir P K 1970 Phys. Rev. D 2540.

[102] Angelopoulos A et al. (CPLEAR collaboration) 1998 Phys. Lett. B 44443.

[103] Wolfenstein L 1999 Int. J. Mod. Phys. E 8501.

[104] Gerber H-J 2004 Eur. Phys. J. C 35195.

[105] Apostolakis A et al. (CPLEAR collaboration) 1999 Phys. Lett. B 456297. 
[106] Bañuls M C, Bernabéu J 1999 Phys. Lett. B 464117.

[107] Lees J P et al. (BABAR collaboration) 2012 Phys. Rev. Lett. 109211801.

[108] Villanueva-Pérez P 2013 J. Phys. Conf. Ser. 447012024.

[109] Bernabeu J et al. 2013 Nucl. Phys. B 868102.

[110] Bernabeu J et al. 2014 Phys. Lett. B 72895.

[111] Hehl F W et al. 2008 Phys. Rev. A 77022106.

[112] Chupp T E et al. 2019 Rev. Mod. Phys. 91015001.

[113] Khriplovich I B, Lamoreaux S 1997 CP violation without strangeness (Berlin, Heidelberg: Springer Verlag).

[114] Purcell E M, Ramsey N F 1950 Phys. Rev. 78807.

[115] Pendlebury J M et al. 2015 Phys. Rev. D 92092003.

[116] Schiff L I 1963 Phys. Rev. 1322194.

[117] Andreev V et al. (ACME collaboration) 2018 Nature 562355.

[118] Regan B C et al. 2002 Phys. Rev. Lett. 88071805.

[119] Graner B et al. 2016 Phys. Rev. Lett. 116161601.

[120] Sahoo B K 2017 Phys. Rev. D 95013002.

[121] Murthy C S et al. 1989 Phys. Rev. Lett. 63965.

[122] Farley F J M et al. 2004 Phys. Rev. Lett. 93052001.

[123] Bennett G W et al. (Muon g-2 collaboration) 2009 Phys. Rev. D 80052008.

[124] Pondrom L et al. 1981 Phys. Rev. D 23814.

[125] Rosendorff S 1960 Nuovo Cim. 17251.

[126] Greaves H, Thomas T 2014 Stud. Hist. Philos. Sci. B 4546.

[127] Bain J 2016 CPT Invariance and the Spin-Statistics Connection (Oxford: Oxford University Press). 
[128] Greenberg O W 2002 Phys. Rev. Lett. 89231602.

[129] Chaichian M et al. 2011 Phys. Lett. B 699177.

[130] Colladay D, Kostelecký V A 1997 Phys. Rev. D 556760.

[131] Chaichian M et al. 2012 Phys. Lett. B 712115.

[132] Fee M S et al. 1993 Phys. Rev. A 48192.

[133] G. Baur et al. (PS210 collaboration) 1996 Phys. Lett. B 368251.

[134] Hori M et al. (ASACUSA collaboration) 2011 Nature 475484.

[135] Ahmadi M et al. (ALPHA collaboration) 2018 Nature 55771.

[136] Gabrielse G et al. (TRAP collaboration) 1999 Phys. Rev. Lett. 823198.

[137] Ulmer S et al. (BASE collaboration) 2015 Nature 524196.

[138] Van Dyck R S, Schwinberg P B, Dehmelt H G 1987 Phys. Rev. Lett. 5926.

[139] Nagahama H et al. (BASE collaboration) 2017 Nature Comm. 8:14084 1.

[140] Chatrchyan S et al. (CMS collaboration) 2017 Phys. Lett. B 77050.

[141] Adamczyk L et al. (STAR collaboration) 2015 Nature 527345.

[142] Adam J et al. (ALICE collaboration) 2015 Nature Physics 11811.

[143] Adam J et al. (STAR collaboration) 2019, preprint arXiv: 1904 . 10520.

[144] Kellerbauer A et al. (AEGIS collaboration) 2008 Nucl. Instr. Meth. B 266 351; Chardin G et al. (GBAR collaboration) 2011 CERN report CERN-SPSC-2011-029, Meyrin; Cronin A D et al. 2009 (AGE collaboration) AGE Letter of Intent, Fermilab 2009, available at Www.fnal.gov/directorate/program_planning/ Mar2009PACPublic/AGELOIFeb2009.pdf.

[145] Kalaydzhyan T 2016 Nature Scient. Rep. 630461.

[146] Amole C et al. (ALPHA collaboration) 2013 Nature Comm. 41785.

[147] Apostolakis A et al. (CPLEAR collaboration) 1999 Phys. Lett. B 452425. 
[148] Anastasi A et al. (KLOE-2 collaboration) 2018 J. High Energ. Phys. 09 021.

[149] Bernabeu J et al. 2015 J. High Energ. Phys. 2015139.

[150] Dolgov A D, Novikov V A 2014 Phys. Lett. B 732244.

[151] Schafer A, Reinhardt J 1995 Phys. Rev. A 51838.

[152] Gonzalez-Garcia M C et al. 2003 Phys. Rev. D 68053007.

[153] de Gouvêa A, Peña-Garay C 2005 Phys. Rev. D 71093002.

[154] Adamson P et al. (MINOS collaboration) 2013 Phys. Rev. Lett. 110251801.

[155] Abe K et al. (T2K collaboration) 2017 Phys. Rev. D $96011102(\mathrm{R})$.

[156] Abe K et al. (Super-Kamiokande collaboration) 2011 Phys. Rev. Lett. 107 241801.

[157] LoSecco J M 1988 Phys. Rev. D 383313.

[158] Sokolowski S M 2017 Acta Phys. Pol. B 481947.

[159] Longo M 2011 Phys. Lett. B 699224.

[160] Shamir L 2012 Phys. Lett. B 71525.

[161] Steigman G 1976 Ann. Rev. Astron. Astrophys. 14339.

[162] Coppi P S 2004, in SLAC Summer Institute on Particle Physics L017.

[163] Adriani O et al. (PAMELA collaboration) 2010 Phys. Rev. Lett. 105 121101; Cuoco A et al. 2017 Phys. Rev. Lett. 118191102.

[164] Agakishiev H et al. (STAR collaboration) 2011 Nature 473353.

[165] Sokol J 2017 Science 356240.

[166] Cohen A G, De Rujula A, Glashow S L 1998 Astrophys. J. 495539.

[167] Krnjaic G 2017 Phys. Rev. D 96035041.

[168] Sakharov A D 1967 JETP Lett. 524. 
[169] 't Hooft G 1976 Phys. Rev. Lett. 378.

[170] Gavela M B et al. 1994 Mod. Phys. Lett. A 9795.

[171] Dimopoulos S, Susskind L 1978 Phys Rev. D 184500.

[172] Cohen A G, Kaplan D B 1987 Phys. Lett. B 199251.

[173] Boyle L et al. 2018 Phys. Rev. Lett. 121251301.

[174] Davidson S et al. 2008 Phys. Rep. 466105.

[175] Carroll S M, Field G B, Jackiw R 1990 Phys. Rev. D 411231.

[176] Lue A, Wang L M, Kamionkowski M 1999 Phys. Rev. Lett. 831506.

[177] Geng C Q et al. 2008 Int. J. Mod. Phys. A 233408.

[178] Pogosian L et al. 2019 Phys. Rev. D 100023507.

[179] Brading K, Castellani E (eds) 2003 Symmetries in Physics: Philosophical Reflections (Cambridge: Cambridge University Press). 